\title{
Long-term Monocular Deprivation during Juvenile Critical Period Disrupts Binocular Integration in Mouse Visual Thalamus
}

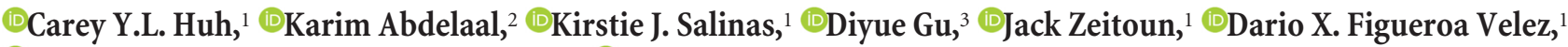 \\ (D) John P. Peach, ${ }^{6}$ Charless C. Fowlkes, ${ }^{4}$ and (D) Sunil P. Gandhi ${ }^{1,5}$ \\ ${ }^{1}$ Department of Neurobiology and Behavior, ${ }^{2}$ School of Biological Sciences, ${ }^{3}$ Donald Bren School of Information \& Computer Sciences, ${ }^{4}$ Department of \\ Computer Science, ${ }^{5}$ Center for Neurobiology of Learning and Memory, University of California, Irvine, California 92697, and ${ }^{6}$ Whiting School of \\ Engineering, Johns Hopkins University, Baltimore, Maryland 21218
}

Study of the neural deficits caused by mismatched binocular vision in early childhood has predominantly focused on circuits in the primary visual cortex (V1). Recent evidence has revealed that neurons in mouse dorsolateral geniculate nucleus (dLGN) can undergo rapid ocular dominance plasticity following monocular deprivation (MD). It remains unclear, however, whether the long-lasting deficits attributed to MD during the critical period originate in the thalamus. Using in vivo two-photon $\mathrm{Ca}^{2+}$ imaging of dLGN afferents in superficial layers of $\mathrm{V} 1$ in female and male mice, we demonstrate that $14 \mathrm{~d}$ MD during the critical period leads to a chronic loss of binocular dLGN inputs while sparing response strength and spatial acuity. Importantly, MD leads to profoundly mismatched visual tuning properties in remaining binocular dLGN afferents. Furthermore, MD impairs binocular modulation, reducing facilitation of responses of both binocular and monocular dLGN inputs during binocular viewing. As predicted by our findings in thalamic inputs, $\mathrm{Ca}^{2+}$ imaging from $\mathrm{V} 1$ neurons revealed spared spatial acuity but impaired binocularity in L4 neurons. V1 L2/3 neurons in contrast displayed deficits in both binocularity and spatial acuity. Our data demonstrate that critical-period MD produces long-lasting disruptions in binocular integration beginning in early binocular circuits in dLGN, whereas spatial acuity deficits first arise from circuits further downstream in V1. Our findings indicate that the development of normal binocular vision and spatial acuity depend upon experience-dependent refinement of distinct stages in the mammalian visual system.

Key words: amblyopia; binocular vision; critical period; dorsolateral geniculate nucleus; thalamus; visual cortex

\section{Significance Statement}

Abnormal binocular vision and reduced acuity are hallmarks of amblyopia, a disorder that affects $2 \%-5 \%$ of the population. It is widely thought that the neural deficits underlying amblyopia begin in the circuits of primary visual cortex. Using in vivo twophoton calcium imaging of thalamocortical axons in mice, we show that depriving one eye of input during a critical period in development chronically impairs binocular integration in thalamic inputs to primary visual cortex. In contrast, visual acuity is spared in thalamic inputs. These findings shed new light on the role for developmental mechanisms in the thalamus in establishing binocular vision and may have critical implications for amblyopia.

\section{Introduction}

The visual system combines information from two eyes to form a coherent, singular view of the world. Visual pathways from each

\footnotetext{
Received July 8, 2019; revised Nov. 6, 2019; accepted Nov. 20, 2019.

Author contributions: C.Y.L.H. and S.P.G. designed research; C.Y.L.H., K.A., K.J.S., and D.X.F.V. performed research; C.Y.L.H., K.A., K.J.S., D.G., J.Z., J.P.P., and C.C.F. analyzed data; C.Y.L.H. wrote the first draft of the paper; C.Y.L.H. and S.P.G. edited the paper; C.Y.L.H. and S.P.G. wrote the paper.

This work was supported by National Institutes of Health DP2 Grant National Eye Institute EY024504 to S.P.G.; and Canadian Institutes of Health Research Postdoctoral Fellowship and Knights Templar Eye Foundation Grants to C.Y.L.H. We thank Karen Bradshaw and Mariyam Habeeb for technical assistance with preliminary experiments; Dennis M. Levi for insightful discussions; and two anonymous reviewers for helpful feedback.
}

eye have been widely thought to remain segregated until the primary visual cortex (V1) where they combine to give rise to binocular vision. Neurons in the visual thalamus have been thought to be predominantly monocular, receiving retinal inputs exclusively from one eye (Casagrande and Boyd, 1996). However, emerging evidence indicates that substantial binocular integra-

The authors declare no competing financial interests.

Correspondence should be addressed to Sunil P. Gandhi at sunil.gandhi@uci.edu or Carey Y. L. Huh at careyhuh@gmail.com.

https://doi.org/10.1523/JNEUROSCl.1626-19.2019

Copyright $\odot 2020$ the authors 
tion occurs in the dorsolateral geniculate nucleus of the thalamus (dLGN). Earlier cat and monkey dLGN studies reported modulation of monocular visual responses by stimulation of the other eye (Marrocco and McClurkin, 1979; Rodieck and Dreher, 1979; Schroeder et al., 1990). Candidate mechanisms for binocular modulation in dLGN include intrathalamic, corticogeniculate, and collicular mechanisms (Dougherty et al., 2019b). More recently, studies in mice and marmosets have revealed both genuine binocular responses and binocularly modulated responses in dLGN (Zhao et al., 2013; Howarth et al., 2014; Zeater et al., 2015; Jaepel et al., 2017). In mice, single dLGN neurons have been shown to receive synaptic inputs from retinal ganglion cells in both eyes, providing an anatomical substrate for direct binocular integration in dLGN (Rompani et al., 2017).

Recently, rapid ocular dominance (OD) plasticity has been demonstrated in the mouse dLGN (Jaepel et al., 2017; Sommeijer et al., 2017), raising the possibility that the thalamus may be implicated in disorders of binocular vision. Abnormal binocular vision and reduced acuity are the hallmarks of amblyopia, a visual disorder that arises from unbalanced binocular input during early childhood (Levi, 2013). Jaepel et al. (2017) showed that short-term (6-8 d) monocular deprivation (MD) leads to increased binocularity in thalamocortical axons in adult mice. However, the effect was transient and required unusual environmental enrichment. Sommeijer et al. (2017) reported that $7 \mathrm{~d}$ MD produced OD plasticity in dLGN in juvenile mice. It remains unclear how these rapid changes in thalamic properties relate to chronic deficits associated with long-term MD, such as impaired spatial acuity and deficits in binocular integration.

Accumulating evidence suggests that the thalamus may be involved in amblyopic deficits. It is well established that, following long-term MD, several functional properties in V1 become chronically disrupted, including OD (Wiesel and Hubel, 1963a; Dräger, 1978; Gordon and Stryker, 1996), spatial acuity (Pizzorusso et al., 2006; Heimel et al., 2007; Bochner et al., 2014), and binocular matching of tuning properties (Movshon and Van Sluyters, 1981; Kaneko and Stryker, 2014; Levine et al., 2017). While early studies using MD models failed to detect functional changes in dLGN neurons (Wiesel and Hubel, 1963b; Blakemore and Vital-Durand, 1986; Levitt et al., 2001), thalamocortical projections have been observed to undergo anatomical changes (Shatz and Stryker, 1978; Antonini and Stryker, 1993; Antonini et al., 1999; Coleman et al., 2010). Impairments of certain dLGN cell types have also been noted in some MD models (Sherman et al., 1972; Duffy et al., 2014). Moreover, brain imaging studies indicate that human amblyopes display anatomical and functional thalamic deficits (Hess et al., 2009; Allen et al., 2015).

To address the question of whether dLGN properties are chronically altered after long-term $\mathrm{MD}$, we used in vivo twophoton $\mathrm{Ca}^{2+}$ imaging to investigate visual response properties of dLGN axons in V1. We found that long-term critical-period MD leads to a profound loss of binocular dLGN afferents while spatial acuity in the thalamocortical pathway is preserved. We also found that MD leads to significant binocular mismatch in remaining binocular dLGN inputs and reduced binocular modulation of both binocular and monocular inputs. V1 L2/3 and L4 neurons also displayed reduced binocularity following $\mathrm{MD}$, but only $\mathrm{L} 2 / 3$ neurons exhibited spatial acuity loss. Together, our findings demonstrate that binocular deficits associated with criticalperiod visual deprivation originate from dLGN inputs, whereas visual acuity deficits arise from downstream cortical circuits.

\section{Materials and Methods}

Animals. For all experiments, mice of both sexes were used. All mice were housed in conventional mouse housing conditions and kept on a $12 \mathrm{~h} / 12$ h light/dark cycle. For thalamocortical axon imaging, we used WT C57BL/6 mice (strain \#027, Charles River) and VGLUT2-Cre mice (Vglut2-ires-cre; stock \#016963, The Jackson Laboratory). VGLUT2-Cre homozygous mice were bred with WT mice to produce heterozygous offspring that were used for imaging. For V1 L2/3 excitatory neuron imaging, a Camk2a-tTa driver line (stock \#007004, The Jackson Laboratory) was crossed to a line expressing GCaMP6s under the control of the tetracycline-responsive regulatory element (tetO; stock \#024742, The Jackson Laboratory) to produce CaMK2a-tTA;tetO-GCaMP6s mice (Wekselblatt et al., 2016); the founder line was heterozygous for both transgenes and maintained by breeding with WT mice. For V1 L4 excitatory neuron imaging, Scnnla-Cre mice (stock \#009613, The Jackson Laboratory) were bred with GCaMP6f reporter mice (Ai93; stock \#024108, The Jackson Laboratory) to create Scnn1a-Ai93 mice heterozygous for both transgenes (Madisen et al., 2010). Mice were weaned at P19 and cohoused with one or more littermate of the same sex until viral injections or cranial window implantation. For all surgeries, body temperature was maintained at $\sim 37.5^{\circ} \mathrm{C}$ by a feedback-controlled heating pad and eyes were covered with ophthalmic ointment to prevent drying. All protocols and procedures followed the guidelines of the Animal Care and Use Committee at the University of California, Irvine.

$M D$. Mice were monocularly deprived (MD) during the critical period for OD plasticity (P19-P33) by eyelid closure (Davis et al., 2015). Under isoflurane anesthesia ( $2 \%$ for induction, $1 \%-1.5 \%$ for maintenance), the nondeprived eye was covered with ophthalmic ointment and the other eye was kept moist with sterile saline. Eye lashes were trimmed and upper and lower eyelids were sutured closed using two mattress sutures (7-0 silk, Ethicon). Eyes were checked every 2-3 d for proper closure. On the 14 th day of MD, the previously closed eye was reopened and carefully checked for any ocular damage under a microscope. If an eye opened prematurely or was found to be damaged, the animal was excluded from the study. Eye health was further monitored for 1-2 weeks following eye reopening.

GCaMP6s virus delivery. For thalamocortical axon imaging, we initially injected AAV1.Syn.GCaMP6s virus into the dLGN of the thalamus in WT C57BL/6 mice, but we found that this approach led to labeling of some V1 cell somata. Thus, we used another approach of injecting AAV1.Syn.Flex.GCaMP6s virus into dLGN in VGLUT2-Cre mice. Since vesicular glutamate transporter 2 (VGLUT2) is predominantly expressed by thalamic neurons (Herzog et al., 2001), we were able to restrict GCaMP6s expression specifically to dLGN neurons using this approach, with little to no cells being labeled in V1. Results from the two approaches were similar, and data from 3 WT and 8 VGLUT2-Cre mice used for functional imaging were combined for analysis. Viral vectors were obtained from Penn Vector Core.

Mice (P58-P80; mean: P67) were placed in a stereotaxic frame under isoflurane anesthesia ( $2 \%$ for induction, $1 \%-1.5 \%$ for maintenance). Mice were injected with lactated Ringer's solution and carprofen (5 mg/ $\mathrm{kg}$, s.c.) for hydration and analgesia. The scalp was retracted, and a small burr hole was made at the injection site using a pneumatic drill. Coordinates used for targeting dLGN were as follows: $\sim 2.2 \mathrm{~mm}$ posterior, $\sim 2.2$ $\mathrm{mm}$ lateral from bregma, and $\sim 2.6 \mathrm{~mm}$ deep from the brain surface. Viral vectors diluted to the final titer of $\sim 1 \times 10^{12} \mathrm{GC} / \mathrm{ml}$ were loaded into a glass pipette and injected into dLGN in one hemisphere (total volume: $80 \mathrm{nl}$, rate: $8 \mathrm{nl} / \mathrm{min}$ ). In MD mice, the hemisphere contralateral to the deprived eye was injected. The skull and injection site were kept moist with saline during the injection. Following surgery, mice were placed on a heat pad to recover and monitored for postoperative health.

Cranial window implantation. Headplate attachment and craniotomy were performed in one surgery following previously reported procedures (Salinas et al., 2017). Briefly, mice were anesthetized with isoflurane (2\% for induction, $1 \%-1.5 \%$ for maintenance), and topical lidocaine (2\%) was applied to provide analgesia. Dexamethasone was administered 2-3 hours before surgery ( $4.8 \mathrm{mg} / \mathrm{kg}$, i.m.). Atropine $(0.15 \mathrm{mg} / \mathrm{kg}$, s.c.) was administered to reduce secretions and aid in respiration. With the head 
secured in a stereotaxic frame, the skull was exposed and an approximate location of binocular V1 (bV1) was marked. A layer of cyanoacrylic glue (3M Vetbond) was applied to the skull, and a custom-printed black headplate was centered over bV1 and fixed to the skull using black dental acrylic (Ortho-Jet, Lang Dental) at an angle parallel to the imaging site. A craniotomy was performed and a No. 1 glass coverslip (4 or $5 \mathrm{~mm}$ in diameter) was placed over the exposed brain and sealed with cyanoacrylic glue and dental acrylic. Mice were placed in a warm cage to recover until mobile and given daily injections of lactated Ringer's and carprofen for at least $3 \mathrm{~d}$ and monitored for postoperative health. In MD mice, craniotomy was performed over bV1 contralateral to the deprived eye.

Widefield imaging for bV1 mapping. Widefield imaging for bV1 mapping was performed through the cranial window after $\geq 4 \mathrm{~d}$ of recovery following craniotomy. For mice used for thalamocortical axon imaging, mapping of bV1 was performed using widefield intrinsic signal imaging, following published procedures (Davis et al., 2015; Salinas et al., 2017). Briefly, awake mice were placed on a smooth platform, head-fixed, and shown contrast-reversing noise stimulus that spanned central $30^{\circ}$ of the mouse's visual field. The stimulus was swept either up or down periodically every $20 \mathrm{~s}$. The stimulus was generated by multiplying a bandlimited $(<0.05 \mathrm{cpd},>2 \mathrm{~Hz}$ ) binarized spatiotemporal noise movie with a one-dimensional Gaussian spatial mask $\left(30^{\circ}\right)$ using custom Python scripts. Visual stimuli were presented on a gamma-corrected 24 inch LED monitor (ASUS VG248, $60 \mathrm{~Hz}$ refresh rate, $20 \mathrm{~cd} / \mathrm{m}^{2}$ mean luminance) at a viewing distance of $25 \mathrm{~cm}$. Widefield fluorescence images were acquired using a SciMedia THT macroscope (Leica PlanApo 1.0 $\times$, $6.5 \times 6.5 \mathrm{~mm}$ imaging area) equipped with an Andor Zyla sCMOS camera. For visualizing vasculature, a green $(530 \mathrm{~nm})$ LED was used. The camera was focused $\sim 600 \mu \mathrm{m}$ beneath the brain surface, located using vasculature, and intrinsic signals were acquired with a red $(617 \mathrm{~nm}) \mathrm{LED}$. The stimulus was presented for 5 min under binocular viewing conditions and typically $2-3$ repeats were run for each condition. Data were analyzed to extract maps of amplitude and phase of cortical responses by Fourier analysis at the frequency of stimulus repetition (Kalatsky and Stryker, 2003) using custom MATLAB (The MathWorks) software. Amplitude was computed by taking the maximum of the Fourier amplitude map smoothed with a $5 \times 5$ Gaussian kernel. For Cam2k-tTA;tetOGCaMP6s and Scnn1a-Ai93 transgenic mice, mapping of bV1 was performed using widefield calcium imaging (blue LED excitation at 465 $\mathrm{nm}$ ), following procedures published previously (Salinas et al., 2017).

In vivo two-photon $\mathrm{Ca}^{2+}$ imaging. All imaging was performed in awake head-fixed mice sitting on a smooth tablet surface. Mice were habituated on the imaging setup for $0.5-1 \mathrm{~h}$ each day for $1-2 \mathrm{~d}$ before imaging. From the same mouse, imaging was performed typically for $2-3 \mathrm{~h}$ per day for $2-5 \mathrm{~d}$ from different field of view (FOV). Ages of mice at imaging were P93-119 (mean: P106) for dLGN axon recordings, P74-P163 (mean: P107) for V1 L2/3 recordings, and P96-P166 (mean: P126) for V1 L4 recordings. The average time interval between GCaMP6s virus injection and two-photon imaging for axon recordings was $39 \mathrm{~d}$.

A resonant two-photon microscope (Neurolabware) and $920 \mathrm{~nm}$ excitation laser (Mai Tai HP, Spectra-Physics) were used for GCaMP6s imaging, following previously published procedures (Salinas et al., 2017) with modifications. A Nikon $16 \times(\mathrm{NA}=0.8)$ water-immersion objective was used. For dLGN bouton imaging, FOV typically covered $\sim 220$ $\mu \mathrm{m} \times 260 \mu \mathrm{m}$, and image sequences were acquired at $8 \mathrm{~Hz}$ (990 lines) at depths of $140 \pm 37 \mu \mathrm{m}$ (mean \pm SD in 37 fields) below the pia, corresponding to cortical layers $1-2 / 3$. Recordings were confined to anterior and middle parts of bV1. For V1 L2/3 excitatory neuron recordings, fields were typically $\sim 700 \mu \mathrm{m} \times 500 \mu \mathrm{m}$, acquired at $7.7 \mathrm{~Hz}$ (1024 lines), and recordings were performed in middle bV1 at cortical depths of $\sim 200 \mu \mathrm{m}$, corresponding to L2/3. For V1 L4 excitatory neuron recordings, fields were typically $\sim 400 \mu \mathrm{m} \times 500 \mu \mathrm{m}$, acquired at $8 \mathrm{~Hz}$ (990 lines), and recordings were performed in middle bV1 at cortical depths of $\sim 390 \mu \mathrm{m}$, corresponding to L4. Data acquisition was controlled by Scanbox software (Neurolabware).

Visual stimuli were generated by custom Python software using PsychoPy 1.8 library. Spherically corrected stimuli were presented on a gamma-corrected 24 inch LED monitor (Asus VG248, $60 \mathrm{~Hz}$ refresh rate,
$20 \mathrm{~cd} / \mathrm{m}^{2}$ ), placed at $25 \mathrm{~cm}$ from the mouse's eyes. The stimuli included full-field drifting sinusoidal gratings (contrast: 99\%) of 5-6 spatial frequencies (SFs; $0.03-0.48$ or $0.03-0.96 \mathrm{cpd}$, spaced logarithmically) and 8 directions $\left(0^{\circ}-315^{\circ}\right.$, in $45^{\circ}$ steps $)$ at a temporal frequency of $2 \mathrm{~Hz}$, a blank (uniform luminance) condition, and a full-field flicker $(2 \mathrm{~Hz})$ condition. Each trial consisted of a visual stimulus for $2 \mathrm{~s}$ and a uniform gray screen for $2 \mathrm{~s}$. Different stimuli were presented in a random order without replacement, and typically 8 repeats were run per stimulus condition. Visual stimuli were presented to one eye at a time, either first to the contralateral or ipsilateral eye using an occluder, and the order of eye presentation was chosen randomly for each session. For binocular viewing experiments, no occluder was used. Eyes were monitored using IRcompatible GigE cameras (Mako-131B, Allied Vision). The illumination by the infrared laser (used for two-photon imaging) was used for pupil tracking.

$\mathrm{Ca}^{2+}$ imaging data analysis. Custom Python software was used to remove motion artifacts, manually identify dLGN boutons and cells, extract fluorescence traces, and perform batch analyses, according to previously described procedures (Salinas et al., 2017) with modifications. We implemented a motion correction algorithm that corrects for translational artifacts by minimizing the Euclidean distance between frames and a template image, using a Fourier transform approach (Dubbs et al., 2016). The outcome of the motion correction was checked by visualizing the mean intensity of 40 pixels in the middle of the frame throughout the movie. To identify regions of interest (ROIs) as boutons or cell bodies, we used the summed intensity projection of the motion-corrected movies and applied morphological criteria to manually identify them.

All pixel values within the ROI region were averaged to yield the fluorescence trace for the ROI. The fluorescence signal of a cell body at time $t$ was determined (Kerlin et al., 2010; Chen et al., 2013) as follows: $F_{\text {cell }}(t)=F_{\text {soma }}(t)-\left(R \cdot F_{\text {neuropil }}(t)\right)$. R was empirically determined to be 0.7 by comparing blood-vessel intensity of GCaMP6s signal with that in the neuropil. The neuropil signal was estimated by taking the mean of the signal in all pixels within $\sim 3 \mu \mathrm{m}$ radius outside the cell's outline. Bouton data were treated to a similar neuropil subtraction, except that for neuropil; a radius of $\sim 1 \mu \mathrm{m}$ outside the bouton's outline was used.

To determine an ROI's response to each stimulus trial, the ROI's trace during the stimulation period was first normalized to the baseline fluorescence value averaged over the $0.5 \mathrm{~s}$ preceding the stimulus $\left(\Delta \mathrm{F} / \mathrm{F}_{0}\right)$. Then, the mean response amplitude (mean $\Delta \mathrm{F} / \mathrm{F}_{0}$ ) was generated for each stimulus type by averaging the normalized response across all trials of that stimulus. An ROI's spontaneous calcium fluctuation was estimated using the ROI's mean response amplitude during blank stimulus presentation. For each SF, an ROI's visual responsiveness was determined using a one-way ANOVA $(p<0.01)$ across responses for all orientations for that SF against responses for the blank condition. For most of the analyses in this paper, we restricted our analyses to ROIs whose responses at the peak SF (SF that gave the strongest response) reached statistical significance at $p<0.01$ (except for data depicted in Fig. $1 F$; see below for Ocular dominance index [ODI] calculation). For V1 L2/3 neuron recordings, an additional criterion was placed such that only cells whose mean $\Delta \mathrm{F} / \mathrm{F}_{0}$ for their preferred stimulus $\left(\mathrm{R}_{\text {pref }}\right)$ was $\geq$ 0.05 were included for further analyses. In Figures $2 C, D, 6 I-K$, and $7 I-K$, we explored whether lowering or raising the significance level to $p<0.05$ or $p<0.005$ affected our results, and we found that the effects of MD remained the same under these different criteria of responsiveness.

For each ROI, the preferred orientation $\left(\theta_{\text {pref }}\right)$ was determined at the ROI's peak SF, by calculating half the mean of the directional vectors weighted by the response $F(\theta)$ at each orientation as follows:

$$
\Theta_{\text {pref }}=\frac{\sum_{i} F\left(\Theta_{i}\right) e^{2 i \Theta_{i}}}{2 \Sigma_{i} F\left(\Theta_{i}\right)}
$$

For each SF, an orientation tuning curve was obtained by fitting a sum of Gaussians function on mean response amplitudes for the eight orientations. The response amplitude at the preferred orientation based on the fitted values was designated as $\mathrm{R}\left(\theta_{\text {pref }}\right)$. To fit a SF tuning curve, response amplitudes at the preferred orientation $\left(\theta_{\text {pref }}\right)$ across the SFs were fitted with a Gaussian function. SF tuning bandwidth was calculated by taking 
the square root of the width at half the maximum of the fit. $R_{\text {pref }}$ is the mean amplitude of the ROI's response to its preferred grating (preferred orientation and SF). For analysis of peak SF values, we first transformed the actual peak SF values $(0.03,0.06, \ldots 0.96 \mathrm{cpd})$ to log-transformed values $(0,1, \ldots 5)$ and performed all statistical analyses on logtransformed values. When reporting summary statistics, the values were transformed back to the actual SF values.

Orientation and direction selectivity (global orientation selectivity index or gOSI, global direction selectivity index or gDSI) for a ROI was determined using a method based on circular variance of the cell's response as follows:

$$
\begin{gathered}
g O S I=\frac{\sqrt{\left(\sum_{i} F\left(\Theta_{i}\right) \sin 2 \Theta_{i}\right)^{2}+\left(\sum_{i} F\left(\Theta_{i}\right) \cos 2 \Theta_{i}\right)^{2}}}{\sum_{i} F\left(\Theta_{i}\right)} \\
g D S I=\frac{\sqrt{\left(\sum_{i} F\left(\Theta_{i}\right) \sin \Theta_{i}\right)^{2}+\left(\sum_{i} F\left(\Theta_{i}\right) \cos \Theta_{i}\right)^{2}}}{\sum_{i} F\left(\Theta_{i}\right)}
\end{gathered}
$$

ODI for each ROI was calculated as $(\mathrm{C}-\mathrm{I}) /(\mathrm{C}+\mathrm{I})$, where $\mathrm{C}$ is $\mathrm{R}_{\text {pref }}$ for contralateral-eye responses and I is $\mathrm{R}_{\text {pref }}$ for ipsilateral-eye responses. In cases where no significant response was detected for one of the eyes according to the responsiveness criteria described above, $R_{\text {pref }}$ for that eye was set to 0 . Thus, responses that were purely driven by the contralateral- versus ipsilateral-eye stimulation were given ODI values of 1 and -1 , respectively. The method of estimating ODI differed for data depicted in Figure $1 F$ only; color coding was based on ODI values calculated according to the same formula as above, except that if one eye did not meet the responsiveness criteria, its $R_{\text {pref }}$ was not set to 0 . Thus, if one of the eyes' responses passed the responsiveness criteria, the other eye's $\mathrm{R}_{\text {pref }}$ was used to calculate ODI in Figure $1 F$.

Histological procedures and anatomical data analysis. After the last imaging session, mice were anesthetized and transcardially perfused with saline and 4\% PFA. Age of mice at perfusion was P112-P142 (mean: P119). Brains were extracted, postfixed, and cryoprotected with $30 \%$ sucrose. The brain was sectioned coronally in $50 \mu \mathrm{m}$ using a frozen sliding microtome (Microm HM450, Thermo Fisher Scientific). Tissue was processed for GFP immunostaining in free-floating sections as follows. Sections were blocked for $1 \mathrm{~h}$ at room temperature with $0.5 \%$ Triton-X (T8787, Millipore Sigma) and 10\% BSA (BP1600-100, Thermo Fisher Scientific) in PBS, then incubated overnight at room temperature with chicken anti-GFP antibody at 1:500 dilution (GFP-1020, Aves Labs). Sections were then washed in PBS and incubated for $2 \mathrm{~h}$ at room temperature with goat anti-chicken IgG antibody tagged with Alexa-488 at 1:1000 dilution (A-11039, Invitrogen). Sections were further processed for nuclear staining (Hoechst 33342), washed in PBS, coverslipped with Flouromount-G (Southern Biotechnology), and imaged.

For dLGN sections, we used an epifluorescence microscope (Carl Zeiss, Axio Imager 2 ) with a $10 \times$ objective. For cell counting, labeled cells in dLGN sections every $200 \mu \mathrm{m}$ (3 sections per animal) were manually counted using the cell counter plugin in Fiji. Total number of labeled dLGN neurons as well as the spatial distribution of labeled neurons in dLGN were quantified for each animal. Functionally imaged brains where post hoc anatomical data revealed that cells were labeled in the neighboring thalamic nucleus LP were excluded from analysis.

For V1 sections, we first took images using the epifluorescence microscope with a $10 \times$ objective. Cortical layers were identified using nuclear staining. To estimate thalamocortical axon density, we obtained the mean fluorescence intensity across the cortical depths in a densely labeled area of a fixed size in V1 $(186 \mu \mathrm{m}$ horizontal $\times 932 \mu \mathrm{m}$ vertical $)$ of each section and quantified labeling intensity in each layer (see Fig. 12D). To get a more accurate estimate of the axon density, we sought to segment axons from the images. For this, $z$-stack images were taken of V 1 sections every $200 \mu \mathrm{m}$ ( 3 sections per animal) using a Carl Zeiss LSM700 confocal microscope and a $20 \times$ objective $(\mathrm{NA}=1.0)$. Images were rotated and cropped to include only superficial layers (L1 and L2/3) in a densely labeled volume of a fixed size in V1 $(100 \mu \mathrm{m}$ horizontal $\times 242 \mu \mathrm{m}$ vertical $\times$ all $z$ slices), which corresponds to the layers that were functionally imaged using in vivo two-photon $\mathrm{Ca}^{2+}$ imaging. Open source neuron tracing software neuTube (Feng et al., 2015) with custom modifications was used to detect axons automatically. The output traces were filtered to remove abnormally large radius nodes, branching points, and isolated nodes. From visual inspection, the auto-segmentation did not detect all visible axons, so the tracing was supplemented by manual tracing by a blinded experimenter. From this final set of traces, we quantified the total axon length per volume and axon radius of thalamocortical axons in V1 L1-2/3 (see Fig. 12 B, E,F; 3 sections per animal).

Statistical analysis. The statistical determination of visual responsiveness is described in detail above; the ANOVA tests for responsiveness, curve-fitting for orientation and SF tuning, and related selectivity/bandwidth calculations were performed in custom Python routines. All other statistical analyses and data plotting were performed using custom software in R. In addition to conventional statistics ( $\chi^{2}$ test, $t$ test, Wilcoxon rank sum test, two- and three-way ANOVA, Kolmogorov-Smirnov test), multilevel statistics were used in some cases to take into account the hierarchical nature of our data (e.g., boutons, neurons, sections nested inside mice). Multilevel linear mixed-effects models with Satterthwaite's approximation were used, with experimental variables (e.g., control vs $\mathrm{MD}$ ) as fixed variables and mouse ID as a random variable. Normality of data was visually checked for and tested using Shapiro-Wilk normality test. If the dataset was deemed non-normal, nonparametric tests (e.g., Wilcoxon rank sum test) were used. For each analysis, the exact statistical test used and sample sizes are described in the figure legends. Only for analysis of number of boutons per field, 1 mouse with an exceptionally large number of dLGN neurons labeled was considered an outlier and excluded from data depicted in Figures $1 H$ and $2 C, D$ (only left panels) but otherwise included in all other analyses and plots. All tests are twotailed. Data are reported as mean \pm SEM unless otherwise noted.

\section{Results}

\section{Long-term critical-period MD leads to a loss of binocular thalamocortical inputs}

To target expression of the calcium sensor GCaMP6s to thalamocortical projections from relay neurons in dLGN, a Cre-dependent GCaMP6s virus was injected into dLGN in VGLUT2-Cre mice (Fig. 1A; see Materials and Methods). We performed calcium imaging in adult mice (P93-119, mean: P106) that were either monocularly deprived for $14 \mathrm{~d}$ during the critical period (P19-P33) or littermate controls (Fig. 1B). Calcium imaging was performed in awake mice that were viewing drifting gratings of various orientations and SFs (Fig. 1C). Twophoton $\mathrm{Ca}^{2+}$ imaging was performed in superficial layers (L12/3) of bV1 (Fig. 1D-G; see also Movie 1). In MD mice, dLGN injections and functional imaging were performed in the hemisphere contralateral to the deprived eye.

Similar to Jaepel et al. (2017), we found a vast majority of dLGN boutons in L1-2/3 to be monocular (visually responsive to contralateral or ipsilateral eye only) with only a small fraction of boutons displaying significant visual responses to both eyes (binocular; $6 \%$ of the population in controls; Fig. $1 \mathrm{I}, \mathrm{J})$. We found that long-term critical-period MD leads to significant reductions in the number of binocular dLGN boutons (69\% reduction) and contralateral (deprived)-eye dominated monocular boutons (23\% reduction) recorded per FOV (Fig. $1 H$ ). The binocular fraction was reduced significantly from $6 \%$ in controls to $3 \%$ in MD mice (Figs. $1 I, J, 2 A$ ).

The loss of binocular dLGN boutons was not due to reduced detectability in MD mice because there was no significant difference in response amplitudes of boutons between control and MD mice (Figs. $1 K, 2 B$ ). Interestingly, binocular boutons displayed approximately twice larger response amplitudes compared with monocular boutons (Figs. $1 K, 2 B$; median $\mathrm{R}_{\text {pref }}$ for binocular boutons: 0.15 , monocular boutons: 0.08 ). The number of virally infected neurons and their spatial distribution in dLGN were 
A

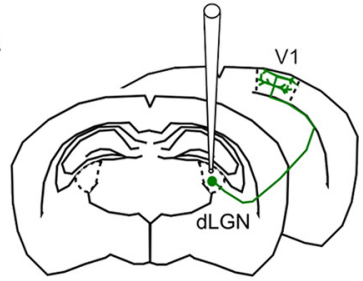

B

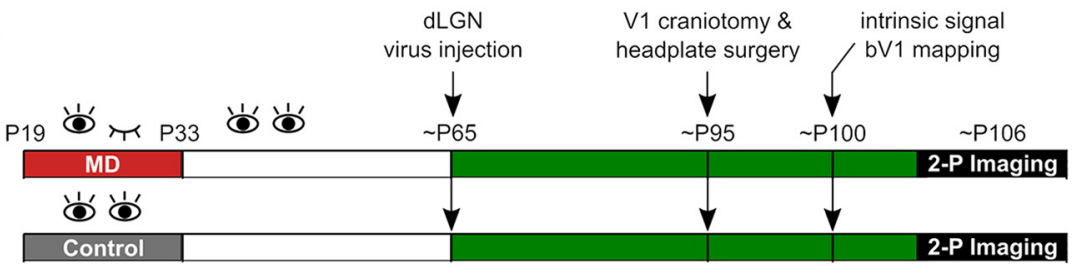

C

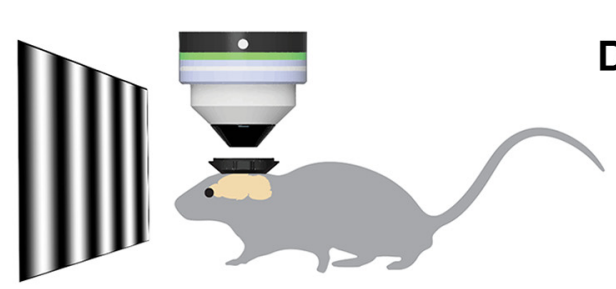

F

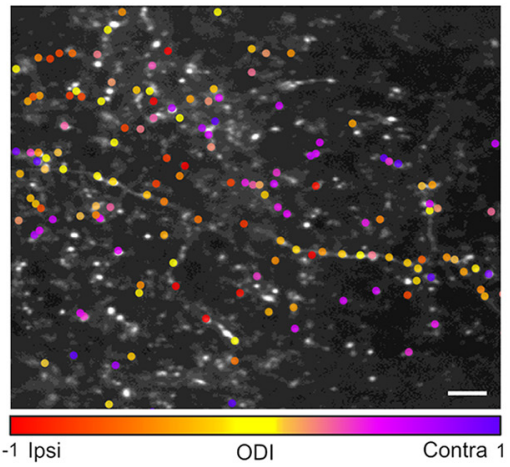

E

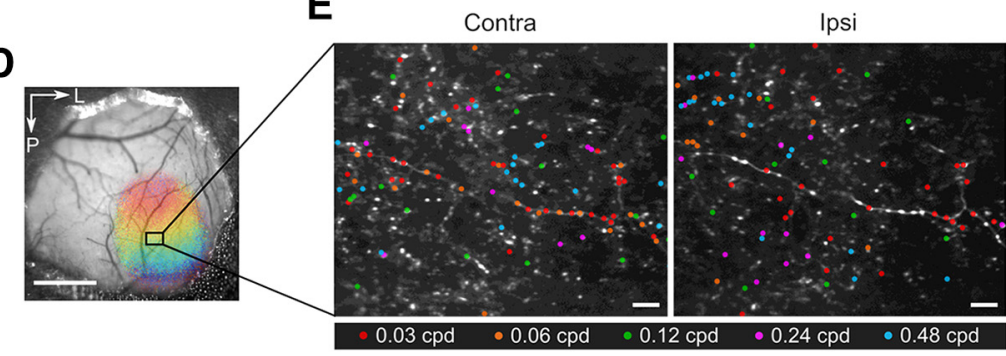

G
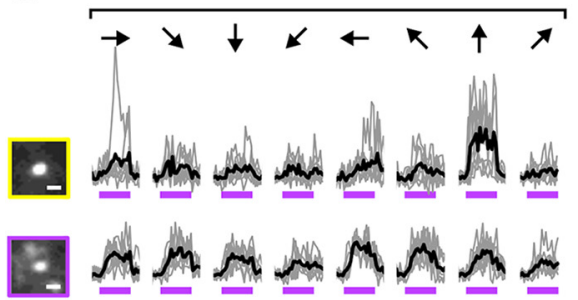

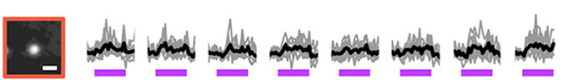

Ipsi
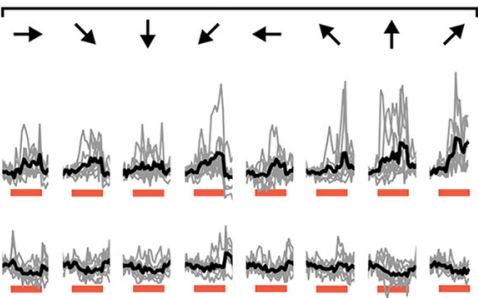

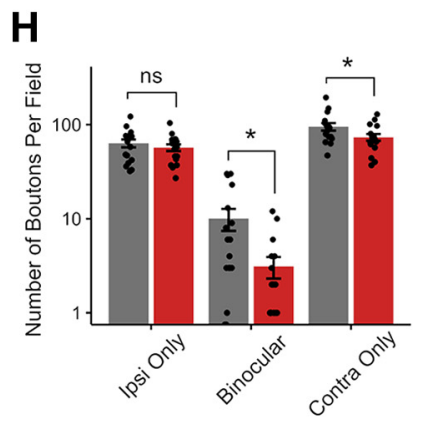

I

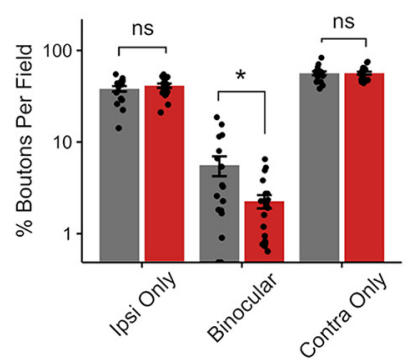

J

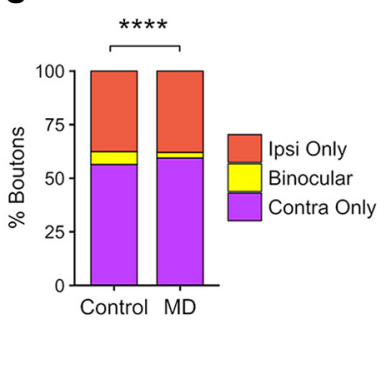

K

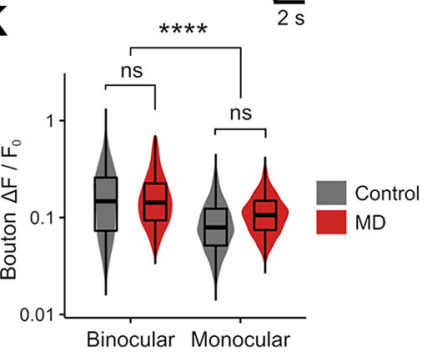

Figure 1. Long-term critical-period MD leads to a loss of binocular thalamocortical inputs. $\boldsymbol{A}$, Schematic of dLGN virus injection, GCaMP6s expression in thalamocortical axons in V1. $\boldsymbol{B}$, Experimental timeline. $\boldsymbol{C}$, In vivo two-photon $\mathrm{Ca}^{2+}$ imaging was performed in awake, head-fixed mice. $\boldsymbol{D}$, An example cranial window with binocular zone mapped using widefield intrinsic signal imaging. Scale bar, $1 \mathrm{~mm}$. E, An example FOV (summed projection) of dLGN boutons imaged in bV1 L1-2/3 of a control mouse. Boutons color-coded according to peak SF during contra-eye (left) and ipsi-eye (right) viewing. Scale bar, $10 \mu \mathrm{m}$. $\boldsymbol{F}$, Same field as in $\boldsymbol{E}$, but color-coded for OD. Scale bar, $10 \mu \mathrm{m} . \mathbf{G}, \mathrm{Ca}^{2+}$ signals in a binocular (top) and two monocular (middle, contra-only; bottom, ipsi-only) boutons in response to drifting gratings presented to contra- or ipsi-eye. Gray represents individual traces. Black represents mean trace. Purple and orange bars represent time of stimulus presentation. Scale bar, $2 \mu \mathrm{m}$. Responses to 8 orientations at peak SF are shown. $\boldsymbol{H}$, Number of visually responsive dLGN boutons that are ipsi-only, binocular, and contra-only per FOV in control versus MD mice (mean \pm SEM per field). Control, ipsi-only: $63 \pm 6$; binocular: $10 \pm 2$; contra-only: $95 \pm 8$ boutons per field. MD, ipsi-only: $57 \pm 4$; binocular: $3 \pm 1$; contra-only: $73 \pm 6$ boutons per field. Linear mixed-effects model, effect of MD for ipsi-only: $F=0.35, p=0.57$; binocular: $F=6.74, p=0.01$; contra-only: $F=4.40, p=0.04 ; n=17$ fields in 5 control mice, 17 fields in 5 MD mice. $I$, Percentage of visually responsive boutons that are ipsi-only, binocular, or contra-only per field in control versus MD mice (mean \pm SEM per field). Control, ipsi-only: $38.1 \pm 2.6 \%$; binocular: $5.6 \pm 1.3 \%$; contra-only: $56.3 \pm 2.6 \%$. MD, ipsi-only: $41.2 \pm 2.1 \%$; binocular: $2.3 \pm 0.4 \%$; contra-only: $56.5 \pm 2.1 \%$. Linear mixed-effects model, effect of MD for ipsi-only: $F=0.23$, $p=0.64$; binocular: $F=6.29, p=0.03$; contra-only: $F=0.02, p=0.90 ; n=17$ fields in 5 control mice, 20 fields in 6 MD mice.J, Ipsi-only, binocular, and contra-only fractions of visually responsive dLGN boutons in control versus MD mice $\left(\chi_{(2)}^{2}=46.96, p=6 \times 10^{-11}\right)$. $K$, Violin and overlaid box plots of response amplitude $R_{\text {pref }}$ of binocular and monocular boutons in control versus $M D$ mice. Linear mixed-effects model, effect of MD: $F=0.29, p=0.59$; binocular versus monocular: $F=229.22, p=2 \times 10^{-16}$. In box plots, middle mark indicates the median, and bottom and top edges indicate 25 th and 75th percentiles, respectively. $\boldsymbol{J}, \boldsymbol{K}, n=2866$ boutons in 5 control mice, 3503 boutons in 6 MD mice. ns, Not significant at $p>0.05$. ${ }^{*} p<0.05,{ }^{* * * *} p<0.0001$.

comparable between functionally imaged control and MD mice and could not account for the reduction of boutons in MD mice (Fig. 3). We also explored whether changing the statistical criteria on visual responsiveness could influence the results, by raising or lowering the significance level to $p<0.05$ or $p<0.005$ (Fig. 2C,D), We found that the effect of MD on
dLGN binocular bouton number per field and binocular proportion remained statistically significant under these different criteria. These findings indicate that long-term critical-period MD leads to a profound loss of binocular thalamocortical inputs without a significant reduction in response strength in the remaining inputs. 


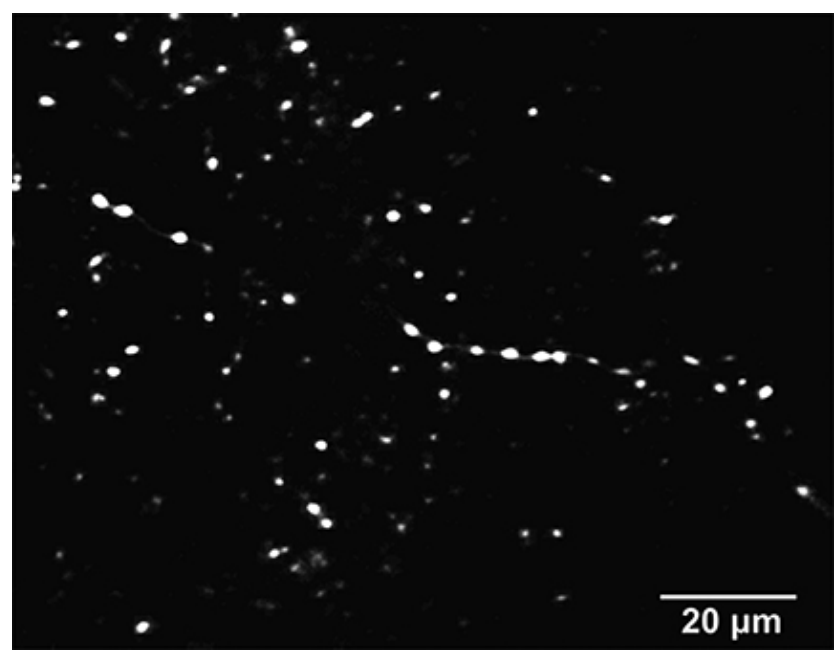

Movie 1. In vivo two-photon $\mathrm{Ca}^{2+}$ imaging of dLGN axons in V1. The movie shows visually evoked activity of $\mathrm{V} 1$ projecting axons from dLGN neurons, visualized using GCaMP6s. The recording shown is from L2/3 of bV1 in a control mouse. During the recording, the mouse was awake, viewing a series of drifting gratings with various spatial frequencies and orientations. The FOV corresponds to the example field shown in Figure

EF, F The axon coursing through the center of the field displayed significant visul both eyes (i.e., binocular).
Intact SF processing in thalamocortical boutons following long-term critical-period MD

Following long-term critical-period MD, mice develop reduced spatial acuity in the deprived eye, a behaviorally demonstrated impairment that lasts well into adulthood (Prusky and Douglas, 2003; Stephany et al., 2014; Davis et al., 2015). It remains unclear whether the acuity deficits are generated in cortical circuits or relayed from dLGN. It is also unknown how SF representation interacts with OD in dLGN. Previously, we have shown that contralateral-eye dominated monocular V1 L2/3 neurons prefer higher SF compared with binocular neurons (Salinas et al., 2017). Thus, we explored how binocularity and SF processing interact in dLGN inputs and how long-term MD affects these properties.

We found that long-term critical-period MD has no significant impact on overall preferred SF of dLGN boutons in V1 L1$2 / 3$ (Fig. $4 E$ ). In both control and MD mice, dLGN boutons were tuned to a wide range of SF (Fig. $4 A-D$ ), and similar percentages of dLGN boutons preferred the highest SFs tested $(0.48-0.96$ cpd) in control versus MD mice (Fig. $4 F$ ). Overall, dLGN boutons were tuned to higher SF compared with V1 L2/3 neurons (Fig. 5A-E), consistent with a previous report using electrophysiological recordings (Durand et al., 2016). Binocular dLGN boutons were tuned to lower SF compared with contralateral-eye dominated monocular boutons, similar to our observations in V1 L2/3 neurons (Figs. 4D-F, 5A-E) (Salinas et al., 2017). SF tuning bandwidths of dLGN boutons were similar between control and MD mice and comparable with those found in V1 neurons (Fig.
A

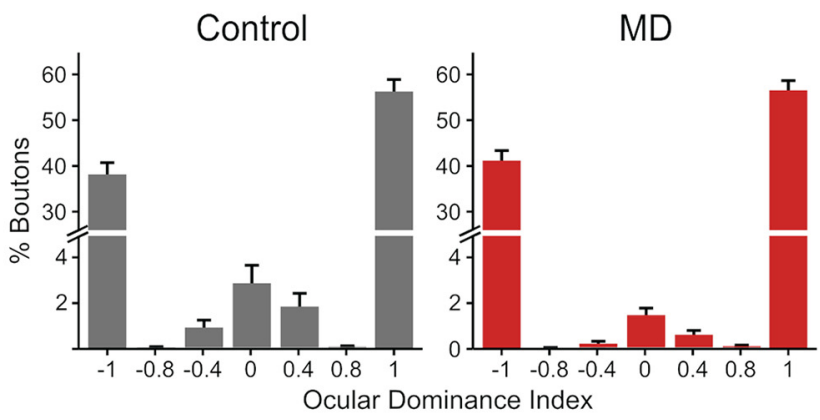

C
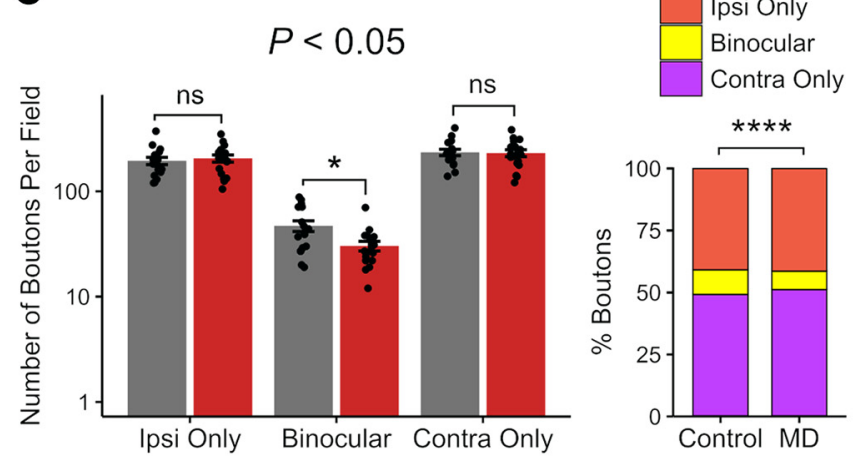

B

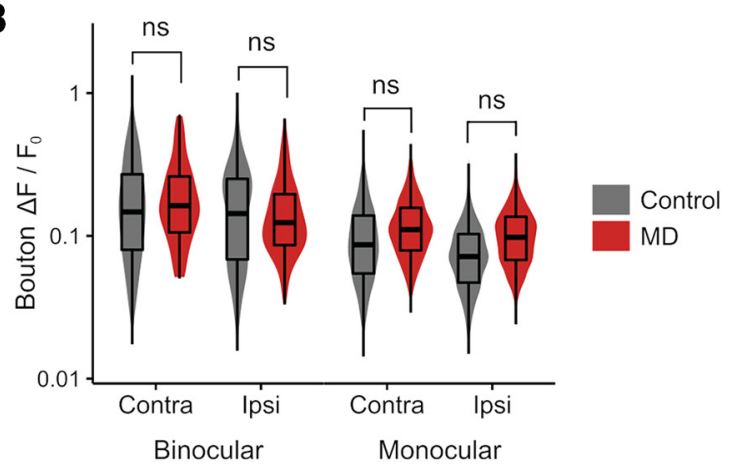

D

$$
P<0.005
$$

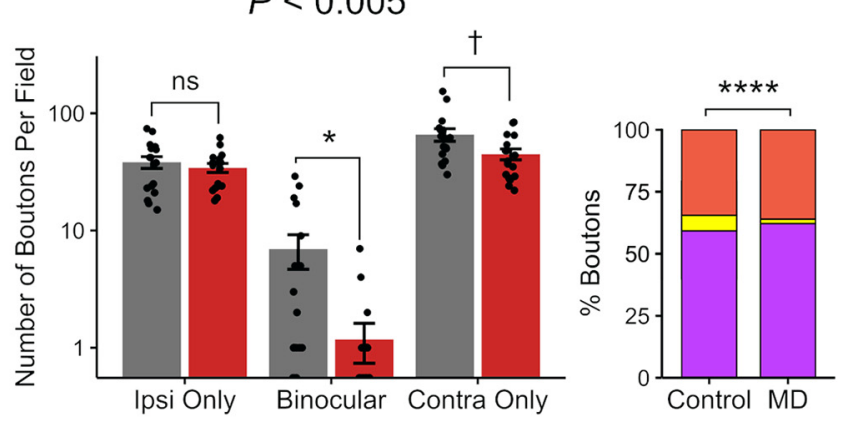

Figure 2. Loss of binocular dLGN boutons without a reduction in response strength. $A, O D$ I histogram of dLGN boutons in control versus MD mice (mean \pm SEM per field, $n=17$ fields in 5 control mice, 20 fields in $6 \mathrm{MD}$ mice). $\boldsymbol{B}$, Violin and overlaid box plots of mean response amplitude $R_{\text {pref }}$ of dLGN boutons in control versus MD mice. Linear mixed-effects model, effect of $M D: F=0.32, p=$ 0.58 ; binocular versus monocular: $F=259.47, p=2 \times 10^{-16}$; contra versus ipsi: $F=44.48, p=2 \times 10^{-11} ; n=2866$ boutons in 5 control mice, 3503 boutons in 6 MD mice. $C, D$, The binocular bouton loss following critical-period MD (Fig. $1 \mathrm{H}, J)$ is shown using two additional statistical criteria in determining visual responsiveness: more liberal $(\boldsymbol{C}: p<0.05)$ or more conservative $(\boldsymbol{D}: p<$ 0.005 ) than the typical criterion used in this study ( $p<0.01$; see Materials and Methods). Linear mixed-effects models, effect of MD for ipsi-only: $p=0.75(\boldsymbol{C}), p=0.55$ (D); for binocular: $p=0.01$ $(C), p=0.01(D)$; for contra-only: $p=0.93(\boldsymbol{C}), p=0.05(\boldsymbol{D})$. $\chi^{2}$ tests: for $C, \chi_{(2)}^{2}=37.50, p=7 \times 10^{-9}, n=8099$ versus 11,028 visually responsive boutons in total, $10 \%$ versus $7 \%$ binocular (control vs MD); for $D, \chi_{(2)}^{2}=54.25, p=1 \times 10^{-12}, n=1887$ versus 2211 visually responsive boutons in total, $6 \%$ versus $2 \%$ binocular (control vs MD). The binocular bouton loss in MD mice remains statistically significant under different data inclusion criteria. In box plots, middle mark indicates the median, and bottom and top edges indicate 25 th and 75 th percentiles, respectively. ns, Not significant at $p>0.1 .{ }^{\dagger} p<0.1,{ }^{*} p<0.05,{ }^{* * * *} p<0.0001$. 
A
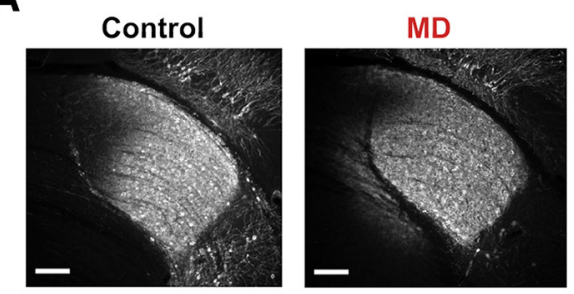

C

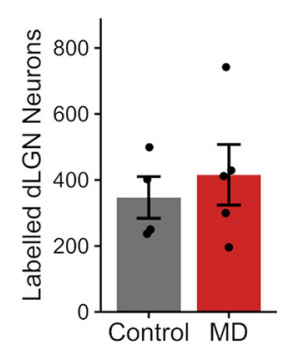

D

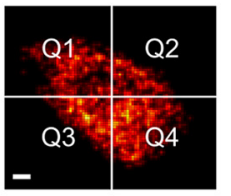

B

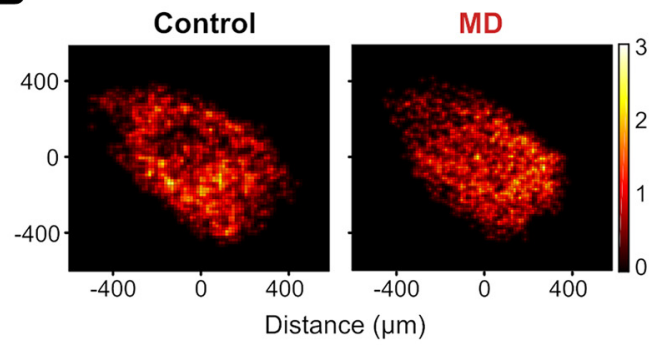

E

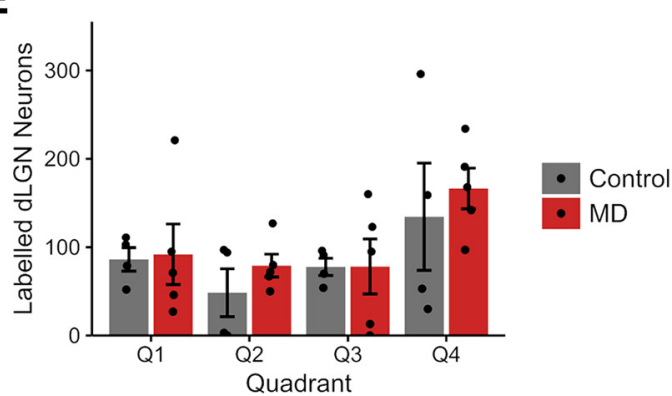

Figure 3. GCaMP6s labeling in $\mathrm{dLGN}$ is comparable between control and MD mice used for functional thalamocortical axon imaging. $A$, Example fluorescence sections of dLGN neurons labeled following GCaMP6s virus injection in control (left) and MD (right) mice that were used for in vivo two-photon $\mathrm{Ca}^{2+}$ thalamocortical axon imaging. Sections were immunostained for GFP. Scale bar, $100 \mu \mathrm{m}$. Example sections in $\boldsymbol{A}$ are from the same mice shown in Figure 12B. B. Heatmaps showing spatial distribution of labeled dLGN neurons in control and MD mice. Heatmaps are based on summed cell counts across all sections and mice. $C$, Numbers of dLGN neurons labeled were similar between functionally imaged control versus MD mice (mean \pm SEM by animal values; $t$ test, $p=$ 0.56). D, Quadrants used in quantifying spatial distribution of labeled dLGN neurons. Scale bar, $100 \mu \mathrm{m}$. $\boldsymbol{E}$, Numbers of labeled dLGN neurons were similar between control and MD mice across all quadrants (mean \pm SEM by animal values; two-way ANOVA, control vs MD: $F=0.65, p=0.43$, effect of quadrant: $F=3.31, p=0.03$, interaction: $F=0.15, p=0.93$ ). $\boldsymbol{B}-\boldsymbol{E}, n=4$ control and $5 \mathrm{MD}$ mice, cells counted from three sections per animal.

$5 F)$. These results indicate that SF processing in dLGN inputs to $\mathrm{V} 1$ remains intact following long-term $\mathrm{MD}$.

\section{In V1 L2/3 neurons, long-term critical-period MD leads to} reductions in binocularity and acuity

To investigate effects on V1 circuits, we examined the impact of long-term critical-period MD on L2/3 excitatory neurons in bV1 by performing two-photon $\mathrm{Ca}^{2+}$ imaging from GCaMP6sexpressing cells in CaMK2a-tTA;tetO-GCaMP6s transgenic mice (Wekselblatt et al., 2016). We found that long-term MD leads to a reduction in the percentage of binocular $\mathrm{L} 2 / 3$ neurons from $29 \%$ to $23 \%$ in control versus MD mice (Fig. $6 A-C$ ) and a significant shift in OD distribution (Fig. 6D). These effects on binocularity occurred without an overall reduction in response amplitude (Fig. 6E). However, in contrast to dLGN boutons, we found that MD leads to a robust reduction in preferred SF of V1 L2/3 neurons, particularly for contralateral (deprived) eye responses (Fig. $6 F, G$ ). Significantly fewer V1 L2/3 neurons preferred $0.48-0.96 \mathrm{cpd}$ in MD mice compared with controls (Fig. $6 H$ ).

Earlier studies, which used dye-loaded calcium recordings in anesthetized mice, reported higher binocular proportions among bV1 L2/3 neurons (Mrsic-Flogel et al., 2007; Kameyama et al., 2010; Scholl et al., 2017) than our results (present study; Salinas et al., 2017). This discrepancy between studies on binocular proportions may reflect differences in sensitivity of the techniques used in expressing calcium indicators, visual stimuli presented, age/state of the animal, and analysis methods used. For example, the previous studies used dye-loaded calcium recordings and low SF (0.03-0.05 cpd) gratings to characterize OD distributions. The high signal-to-noise GCaMP6 recordings and the wide range of SFs used in our current study and in Salinas et al. (2017) $(0.03-0.96 \mathrm{cpd})$ may have allowed us to pick up responses that the other studies missed, including weaker monocular responses evoked by higher SFs. Indeed, we found that visually evoked re- sponses in monocular neurons are overall smaller in amplitude compared with binocular neurons (Fig. 6E), and neurons that are responsive to the highest SFs $(0.48-0.96 \mathrm{cpd})$ are mostly monocular (Fig. 6H) (Salinas et al., 2017). To test the potential influence of statistical criteria, we applied a more liberal statistical criterion on visual responsiveness $(p<0.05)$. Overall, we found more binocularly responsive V1 L2/3 neurons, but the effects of MD on binocular fraction and spatial acuity stayed consistent (Fig. 6I$K)$. These findings demonstrate that long-term MD impacts the development of both OD and spatial acuity in V1 L2/3 neurons.

In V1 L4 neurons, long-term critical-period MD leads to reduced binocularity but preserved spatial acuity

We also examined the impact of long-term MD on V1 L4 neurons using Cre-driven GCaMP6f expression in Scnn la-Cre mice. Similar to dLGN boutons, we found that the binocular fraction among V1 L4 neurons was reduced from $9 \%$ to $4 \%$ in control versus $\mathrm{MD}$ mice (Fig. $7 A-D$ ). Long-term $\mathrm{MD}$ did not significantly impact V1 L4 neurons' overall response strength (Fig. 7E) or their SF processing (Fig. $7 F-H$ ). V1 L4 neurons' mean peak SF and the percentage of V1 L4 neurons preferring $0.48-0.96 \mathrm{cpd}$ were similar in control versus MD mice (Fig. $7 G, H$ ). Making the statistical criterion on responsiveness more liberal $(p<0.05)$ increased the binocular fraction among V1 L4 neurons but did not change the effects of MD (Fig. $7 I-K$ ).

Recent studies have investigated V1 L4 neurons' properties in terms of orientation/direction selectivity (e.g., Sun et al., 2016) and found L4 neurons' properties to be somewhere in between properties of dLGN boutons and L2/3 neurons, which is similar to what we report here in terms of L4 neurons' SF and OD properties (Figs. 5A-E, 7D). We found that, in V1 L4 neurons, binocularity was altered in MD mice, whereas acuity was not (Fig. 7), similar to the changes observed in dLGN boutons (Figs. 1, 2, 4). Indeed, several previous studies have shown that V1 L4 and L2/3 neu- 
A

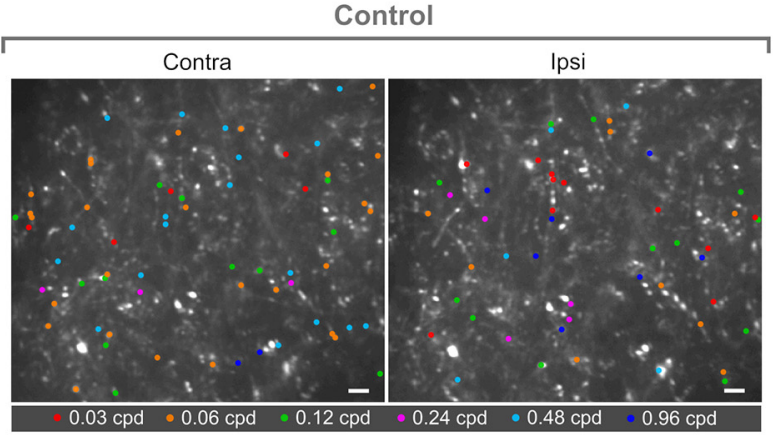

B

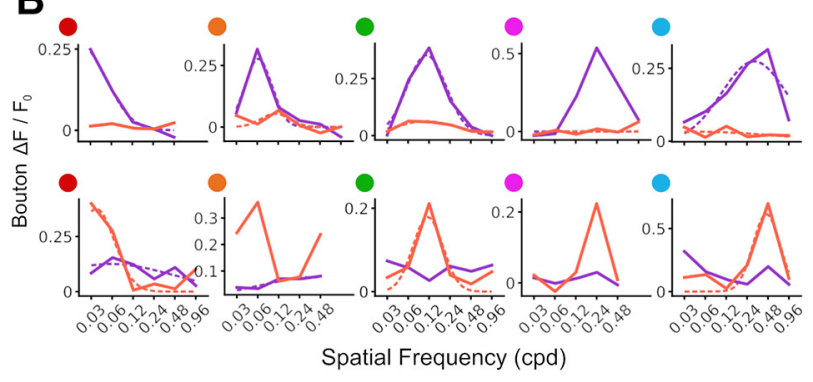

D

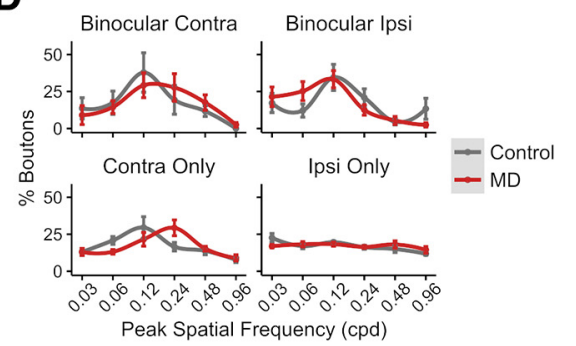

${ }^{c}$
E

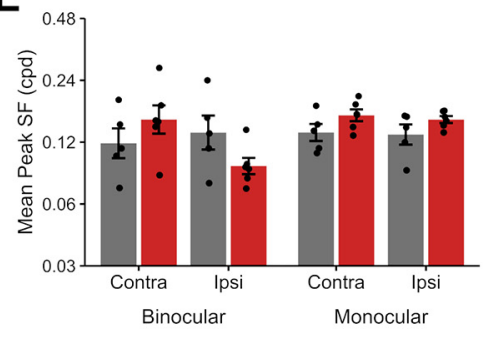

MD
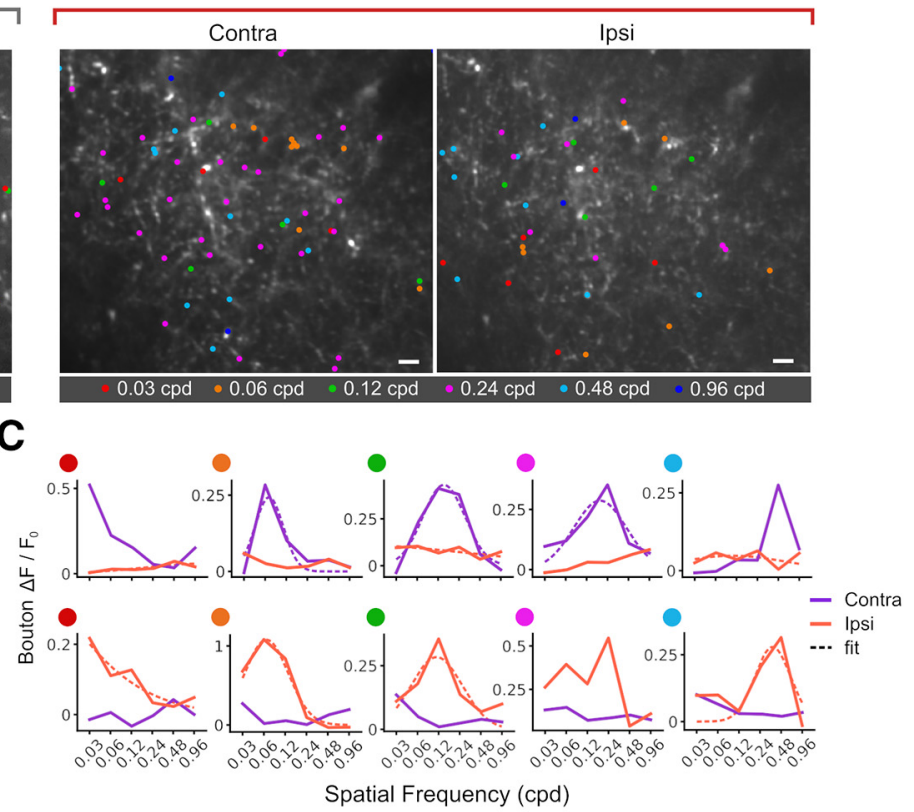

F

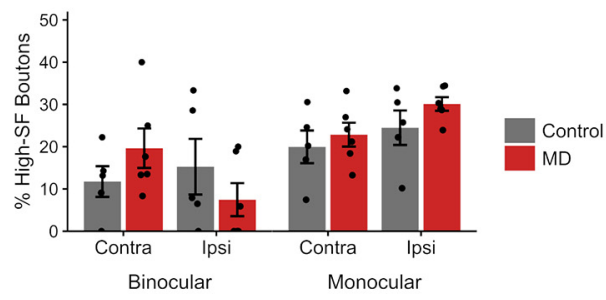

Figure 4. Intact SF processing in thalamocortical boutons following long-term critical-period MD. $A$, Example FOVs of dLGN boutons imaged in bV1, color-coded according to peak SF of bouton during contralateral- and ipsilateral-eye presentation in control versus MD mice. Scale bar, $10 \mu \mathrm{m}$. $\boldsymbol{B}$, Example SF tuning curves of monocular (top row, contra-only; bottom row, ipsi-only) boutons in control mice. Purple represents contralateral-eye trials. Orange represents ipsilateral-eye trials. Solid lines indicate mean response amplitudes. Dotted lines indicate fitted curves based on mean values. Fits omitted if curve-fitting failed to merge. C, Example SF tuning curves of monocular boutons in MD mice. Same convention as in $\boldsymbol{B}$. D, Mean probability distribution of peak SF in binocular (top) and monocular (bottom) dLGN boutons' contralateral- (left) and ipsilateral-eye (right) responses (mean \pm SEM by animal values). Mean values were fitted with a local regression smoothing function. $E$, Mean peak SF of boutons in control versus MD mice (mean \pm SEM by animal; three-way ANOVA, control vs MD: $F=0.54, p=0.47$, binocular vs monocular: $F=4.32, p=0.04$, contra vs ipsi: $F=2.42, p=0.13$ ). $F$, Percentage of boutons with peak SF of $0.48-0.96$ cpd ("High-SF boutons"; mean \pm SEM by animal values; three-way ANOVA, effect of MD: $F=0.57, p=0.46$, binocular vs monocular: $F=15.05, p=0.004$, contra vs ipsi: $F=0.03, p=0.87) . \boldsymbol{D}-\boldsymbol{F}, n=2866$ boutons in 5 control mice, 3503 boutons in $6 \mathrm{MD}$ mice.

rons are quite distinct in terms of integration of thalamic inputs and plasticity mechanisms (Daw et al., 1992; Trachtenberg et al., 2000; Morgenstern et al., 2016), in line with our results.

Together, these findings indicate that MD-induced binocularity deficits observed at the level of V1 neurons may originate, at least in part, from the binocularity loss in dLGN because binocularity deficits are evident as early as in dLGN boutons and are propagated to V1 L4 and L2/3 neurons. In contrast, SF processing is intact at the level of thalamocortical inputs from dLGN and in V1 L4 neurons; thus, MD-induced impairments in spatial acuity first emerge in V1 L2/3 neurons.

\section{Binocular mismatch in thalamocortical boutons following long-term critical-period MD}

V1 neurons have been shown to display a significant binocular mismatch in orientation tuning following long-term critical-period MD (Wang et al., 2010; Levine et al., 2017), yet whether the mismatch originates from dLGN inputs has been unclear. In addition, it remains unknown whether binocular mismatch extends to other vi- sual properties, such as SF tuning. Thus, we investigated whether MD leads to binocular mismatch in dLGN afferents in terms of response amplitude, preferred SF, and orientation.

In controls, binocular dLGN boutons were found to be exquisitely well matched between the eyes in terms of response amplitude, preferred SF, and orientation/direction (Fig. 8). The majority $(51 \%)$ of binocular boutons displayed ODI values between -0.2 and 0.2 (Fig. $8 B-D$ ), indicating well-matched response amplitudes between the eyes. Many binocular boutons $(41 \%)$ showed exact peak-SF matching between the eyes (Fig. $8 E-G)$. If boutons were mismatched in terms of SF tuning, preferred SF was higher in contralateral- or ipsilateral-eye responses in approximately equal proportions of boutons in control mice (Fig. $8 E, G$ ). There was also significant binocular matching in terms of preferred orientation among orientation/directionselective binocular boutons (Fig. $8 H-J$ ).

We found that long-term MD leads to profound binocular mismatch in multiple visual response properties. In MD mice, binocular boutons were significantly more mismatched in response amplitude 
A dLGN Boutons

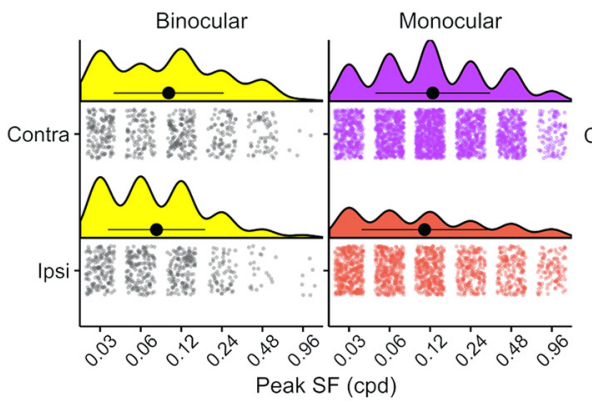

D

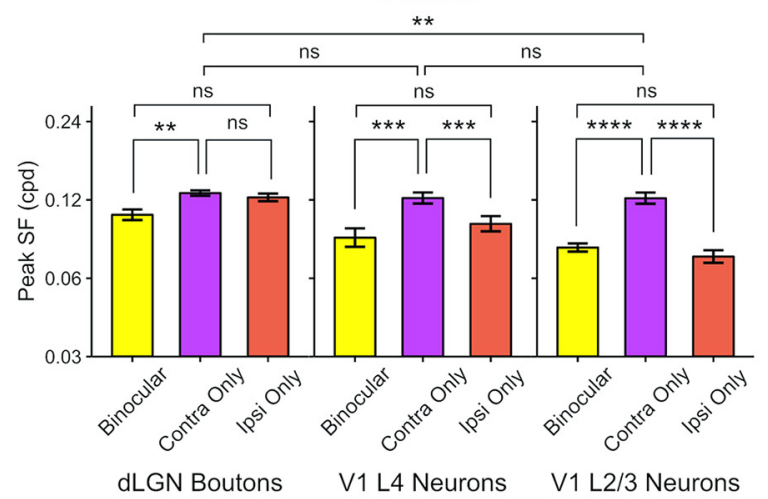

V1 L4 Neurons

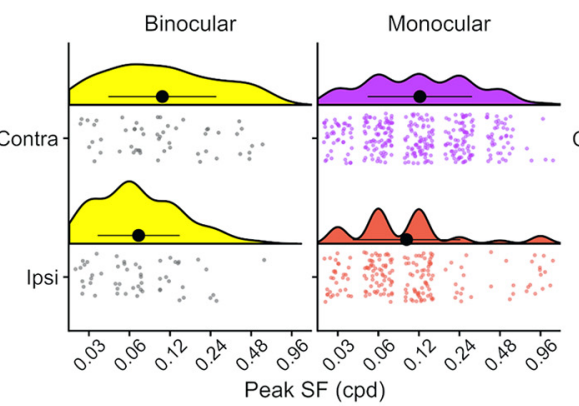

C

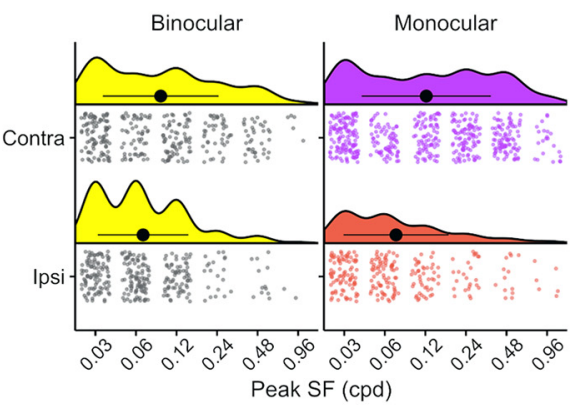

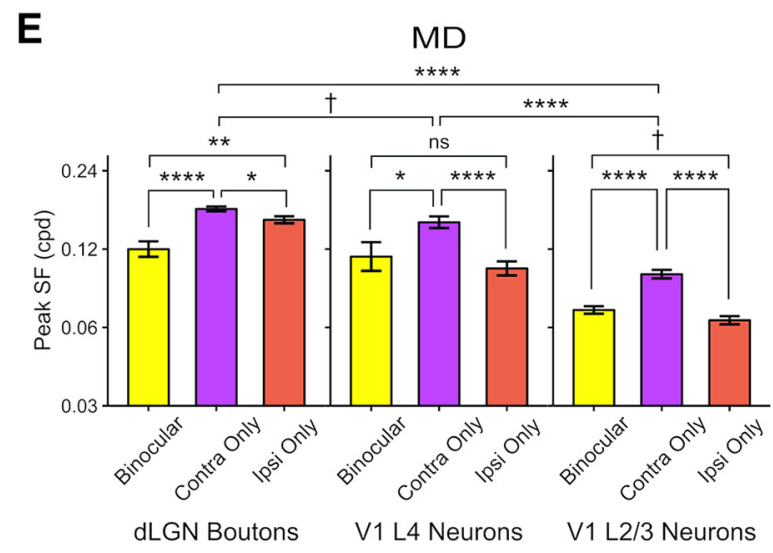

$\mathbf{F}$ F dLGN Boutons V1 L4 Neurons V1 L2/3 Neurons
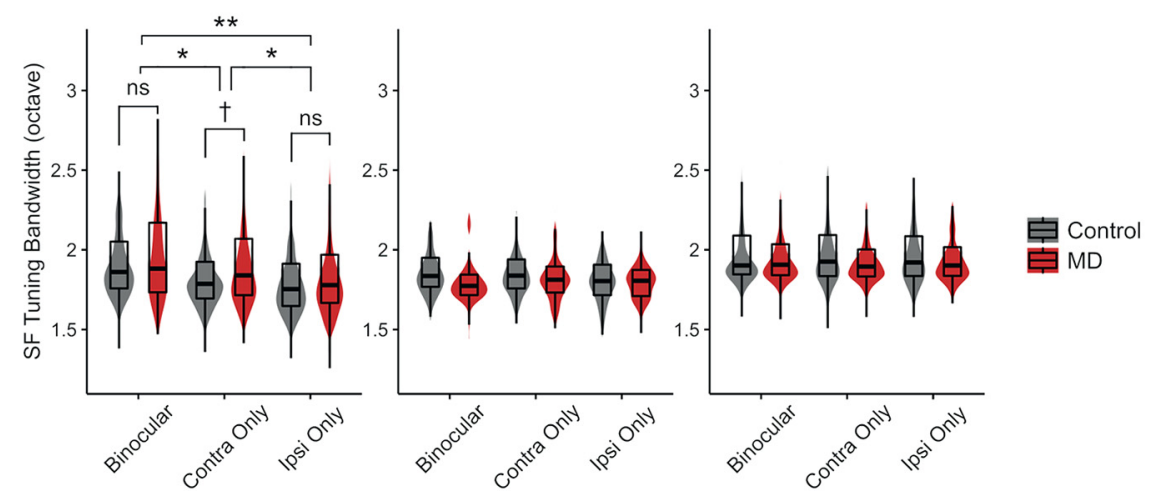

Figure 5. Comparison of SF tuning properties of dLGN boutons, V1 L4 and L2/3 neurons. $A-C$, Control data only. $A$, Raincloud plots represent distributions of peak SF in binocular (left) and monocular (right) (purple represents contra-only; orange represents ipsi-only) dLGN boutons during contralateral- (top) and ipsilateral-eye (bottom) visual stimulation. Individual data points are plotted jittered to avoid overplotting. Black filled circles and lines represent mean \pm SD. B, Distribution of peak SF in V1 L4 neurons. C, Distribution of peak SF in V1 L2/3 neurons. D, Mean peak SF (mean \pm SEM of all sample) of binocular, contra-only, and ipsi-only dLGN boutons (left), V1 L4 neurons (middle), and V1 L2/3 neurons (right) in control mice. Linear mixed-effects models. Boutons versus $L 4$ neurons: $F=2.37, p=0.16$; boutons versus $L 2 / 3$ neurons: $F=10.96, p=0.004 ; L 4$ versus $L 2 / 3$ neurons: $F=0.06, p=0.80$; binocular versus contra-only boutons: $F=7.94, p=0.004$; binocular versus ipsi-only boutons: $F=1.98, p=0.15$; contra-only versus ipsi-only boutons: $F=2.29, p=0.12$; binocular versus contra-only $L 4$ neurons: $F=11.83, p=0.0006$; binocular versus ipsi-only $L 4$ neurons: $F=0.001, p=0.96$; contra-only versus ipsi-only $L 4$ neurons: $F=13.46, p=0.0002$; binocular versus contra-only $L 2 / 3$ neurons: $F=42.71, p=9 \times 10^{-11}$; binocular versus ipsi-only L2/3 neurons: $F=1.54, p=0.21$; contra-only versus ipsi-only L2/3 neurons: $F=38.33, p=9 \times 10^{-10} . E$, Mean peak SF ( \pm SEM) of binocular, contra-only, and ipsi-only dLGN boutons and $V 1 L 4, L 2 / 3$ neurons in MD mice (same convention as $D$ ). Linear mixed-effects models. Boutons versus $L 4$ neurons: $F=4.26, p=0.05 ;$ boutons versus $L 2 / 3$ neurons: $F=130.63, p=3 \times$ $10^{-10} ; \mathrm{L} 4$ versus $L 2 / 3$ neurons: $F=58.09, p=1 \times 10^{-6}$; binocular versus contra-only boutons: $F=24.37, p=8 \times 10^{-7}$; binocular versus ipsi-only boutons: $F=8.61, p=0.003 ;$ contra-only versus ipsi-only boutons: $F=4.90, p=0.02$; binocular versus contra-only $L 4$ neurons: $F=3.91, p=0.04$; binocular versus ipsi-only $L 4$ neurons: $F=0.50, p=0.47$; contra-only versus ipsi-only L4 neurons: $F=24.67, p=9 \times 10^{-7}$; binocular versus contra-only L2/3 neurons: $F=40.21, p=3 \times 10^{-10}$; binocular versus ipsi-only L2/3 neurons: $F=3.37, p=0.06 ;$ contra-only versus ipsi-only L2/3 neurons: $F=52.40, p=8 \times 10^{-13} . F$, Violin and overlaid box plots of SF tuning bandwidth in binocular, contra-only, and ipsi-only dLGN boutons (left), V1 L4 neurons (middle), and V1 L2/3 neurons (right) in control versus MD mice. Linear mixed-effects models. Boutons: effect of $M D: F=2.65, p=0.12$; effect of eye group: $F=4.42, p=0.01$. Post hoc tests, binocular versus contra-only: $F=4.09, p=0.04$; binocular versus ipsi-only: $F=7.49, p=0.006$; contra-only versus ipsi-only: $F=4.02, p=0.04$. V1 L4 neurons: effect of $M D: F=1.04, p=0.30 ;$ effect of eye group: $F=0.72, p=0.48$. V1 L2/3 neurons: effect of $\mathrm{MD}: F=0.54, p=0.47$; effect of eye group: $F=0.42, p=0.65$. In box plots, middle mark indicates the median, and bottom and top edges indicate 25th and 75th percentiles, respectively. All panels: $n=2866$ boutons in 5 control mice, 3503 boutons in 6 MD mice; 572 L4 neurons in 3 control mice, 565 L4 neurons in 2 MD mice; 1051 L2/3 neurons in 9 control mice, and $1355 \mathrm{~L} 2 / 3$ neurons in 6 MD mice. ns, Not significant at $p>0.1 .{ }^{\dagger} p<0.1,{ }^{*} p<0.05,{ }^{* *} p<0.01,{ }^{* * *} p<0.001,{ }^{* * * *} p<0.0001$. 
A
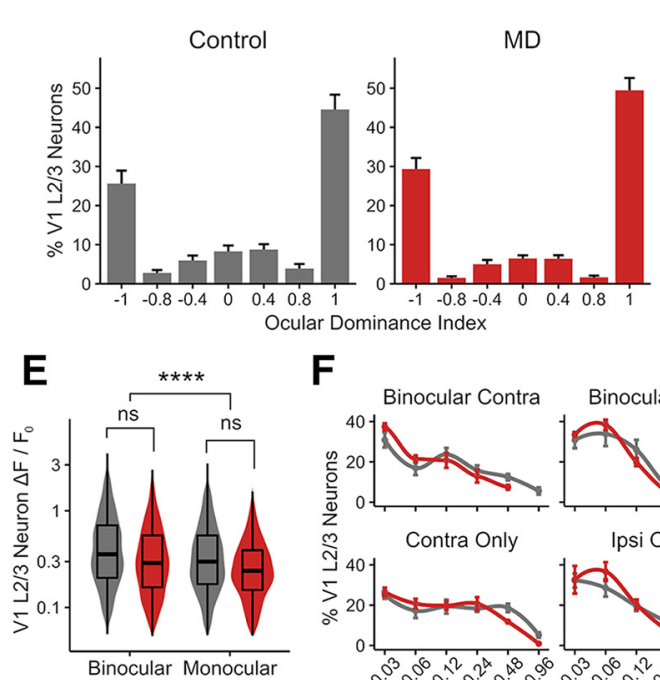

$F$

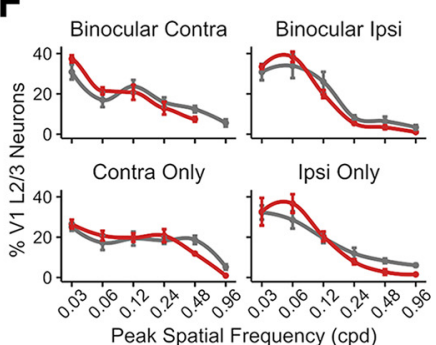

B

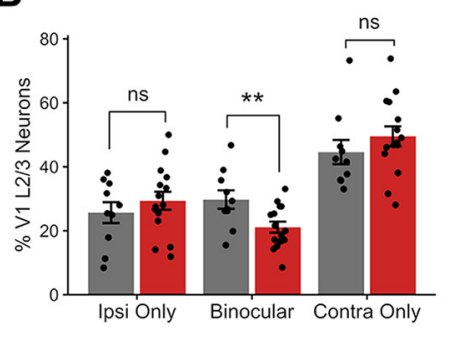

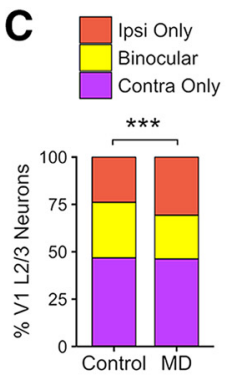

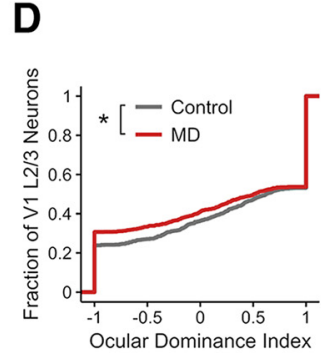

G
H

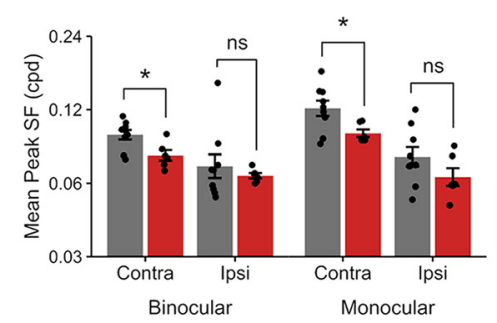

K

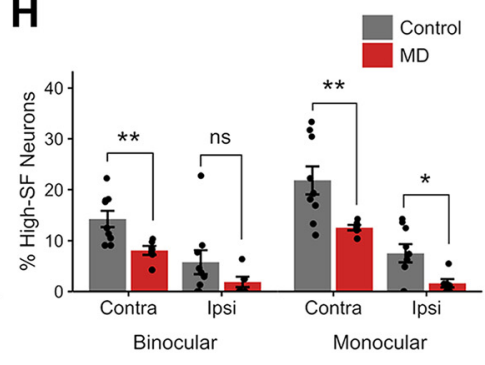

I $P<0.05$

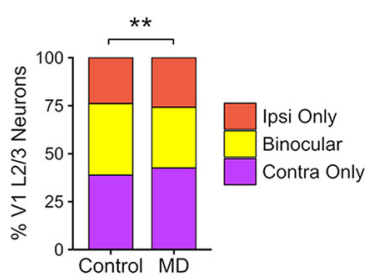

J

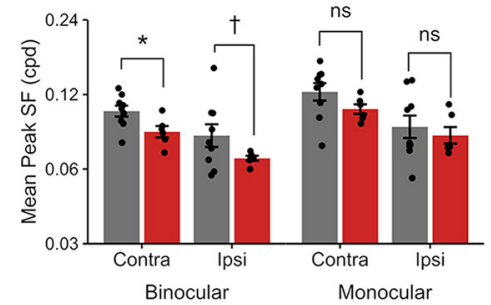

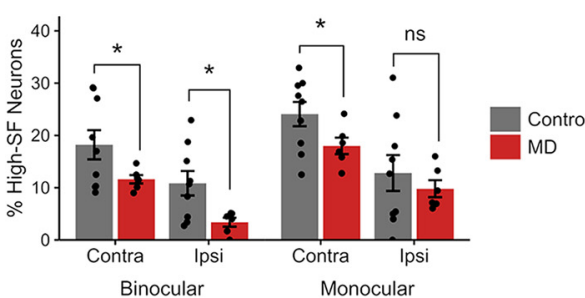

Figure 6. In V1 L2/3 neurons, long-term critical-period MD leads to reductions in binocularity and spatial acuity. $A, 0$ DI histogram of V1 L2/3 neurons in control versus MD mice (mean \pm SEM per field, $n=10$ fields in 9 control mice, 15 fields in $6 \mathrm{MD}$ mice). $\boldsymbol{B}$, Percentage of visually responsive V1 L2/3 excitatory neurons that are ipsi-only, binocular, and contra-only per field in control versus MD mice (mean \pm SEM per field). Linear mixed-effects model, effect of MD for ipsi-only: $F=0.78, p=0.38$; binocular: $F=8.09, p=0.008$; contra-only: $F=1.09, p=0.30$. C, Ipsi-only, binocular, and contra-only fractions of visually responsive V1 L2/3 neurons in control versus $\operatorname{MD}$ mice $\left(\chi_{(2)}^{2}=17.42, p=0.0001\right)$. $D$, Cumulative distribution of $0 D$ I values from V1 L2/3 neurons in control versus MD mice (-10DI: ipsi-only; 1: contra-only; Kolmogorov-Smirnov test, $p=0.01$ ). E, Violin and overlaid box plots of response amplitude $R_{\text {pref }}$ of binocular and monocular V1 L2/3 neurons in control versus $M D$ mice. Linear mixed-effects model, effect of $M D: F=1.30, p=0.27$; binocular versus monocular: $F=59.43, p=1 \times 10^{-14}$. In box plots, middle mark indicates the median, and bottom and top edges indicate 25 th and 75 th percentiles, respectively. $F$, Mean probability distribution of peak SF in binocular (top) and monocular (bottom) V1 L2/3 neurons' contralateral- (left) and ipsilateral-eye (right) responses (mean \pm SEM by animal values). There is a leftward shift of SF distribution curves in MD mice compared with controls. Mean values were fitted with a local regression smoothing function. G, Mean peak SF of V1 L2/3 neurons in control versus MD mice (mean \pm SEM by animal values). Three-way ANOVA, effect of MD: $F=10.03, p=0.002$; binocular versus monocular: $F=6.53, p=0.01$; contra versus ipsi: $F=39.82, p=6 \times 10^{-8}$. Post hoc tests, effect of MD in binocular-contra: $p=0.01 ;$ binocular-ipsi: $p=0.45$; monocular-contra: $p=0.01$; monocular-ipsi: $p=0.14$. H, Percentage of V1 L2/3 neurons with peak SF of $0.48-0.96 \mathrm{cpd}$ (high-SF neurons; mean \pm SEM by animal values). Three-way ANOVA, effect of MD: $F=20.79, p=3 \times 10^{-5}$; binocular versus monocular: $F=7.21, p=0.009$; contra versus ipsi: $F=57.40, p=5 \times 10^{-10}$. Post hoc tests, effect of MD in binocular-contra: $p=0.005$; binocular-ipsi: $p=0.15$; monocular-contra: $p=0.009$; monocular-ipsi: $p=0.01 . \boldsymbol{A}-\boldsymbol{H}, n=1051$ neurons in 9 control mice, 1355 neurons in 6 MD mice. $\boldsymbol{I}-\boldsymbol{K}$, The decrease in binocular fraction and acuity deficits following critical-period $\operatorname{MD}(\boldsymbol{C}, \boldsymbol{G}, \boldsymbol{H})$ is shown using a more liberal statistical criterion $(p<0.05)$ in determining visual responsiveness than the typical criterion used in this study $\left(p<0.01\right.$; see Materials and Methods). $I$, Same convention as in $C . \chi_{(2)}^{2}=10.95, p=0.004 ; 37 \%$ versus $31 \%$ binocular (control vs MD). J, Same convention as in G. Three-way ANOVA, effect of MD: $F=7.48, p=0.008$; binocular versus monocular: $F=8.03, p=0.006$; contra versus ipsi: $F=21.03, p=$ $2 \times 10^{-5}$. Post hoc tests, effect of MD in binocular-contra: $p=0.02$; binocular-ipsi: $p=0.08$; monocular-contra: $p=0.11$; monocular-ipsi: $p=0.55$. $\boldsymbol{K}$, Same convention as in $\boldsymbol{H}$. Three-way ANOVA, effect of MD: $F=10.50, p=0.002$; binocular versus monocular: $F=7.87, p=0.007$; contra versus ipsi: $F=25.75, p=5 \times 10^{-6}$. Post hoc tests, effect of MD in binocular-contra: $p=0.04$; binocular-ipsi: $p=0.01$; monocular-contra: $p=0.05$; monocular-ipsi: $p=0.44 . \boldsymbol{I}-\boldsymbol{K}, n=1332$ neurons in 9 control mice, 1881 neurons in 6 MD mice. ns, Not significant at $p>0.1 .{ }^{\dagger} p<0.1,{ }^{*} p<0.05,{ }^{* *} p<0.01,{ }^{* * *} p<0.001,{ }^{* * * *} p<0.0001$.

between the eyes compared with controls, resulting in a small but significant shift of ODI toward the contralateral (deprived) eye (ODI shift: 0.07 ; Fig. $8 C, D$ ). We also found marked binocular mismatch in preferred SF, with ipsilateral (non-deprived) eye responses being tuned to lower SF compared with contralateral-eye responses in MD mice (Fig. $8 E-G$ ). The SF mismatch was not due to binocular boutons exhibiting broader SF tuning in MD mice (Fig. $5 F$ ). Long-term $\mathrm{MD}$ was also found to lead to a significant mismatch in preferred orientation in orientation/direction-selective binocular dLGN boutons (Fig. $8 H-J$ ). Overall, many dLGN boutons were found to be highly direction-selective (Figs. $8 A, 9 A, B$ ), consistent with previously reported properties of dLGN neurons projecting to superficial layers of V1 (Marshel et al., 2012; Piscopo et al., 2013; Cruz-Martín et al., 2014; Kondo and Ohki, 2016; Roth et al., 2016; Sun et al., 2016). Orientation/direction selectivity indices were similar between control and MD mice (Fig. 9C,D). These findings indicate that normal binocular experience during the critical period is necessary for proper binocular matching of dLGN inputs' visual properties, including response amplitude, SF, and orientation/direction tuning.

\section{Long-term critical-period MD impairs binocular facilitation} of thalamocortical boutons

Neurons in the mouse dLGN display various types of binocular modulation (modulation of activity during binocular viewing compared with monocular viewing), including facilitation and suppression (Zhao et al., 2013; Howarth et al., 2014). We investigated 
A

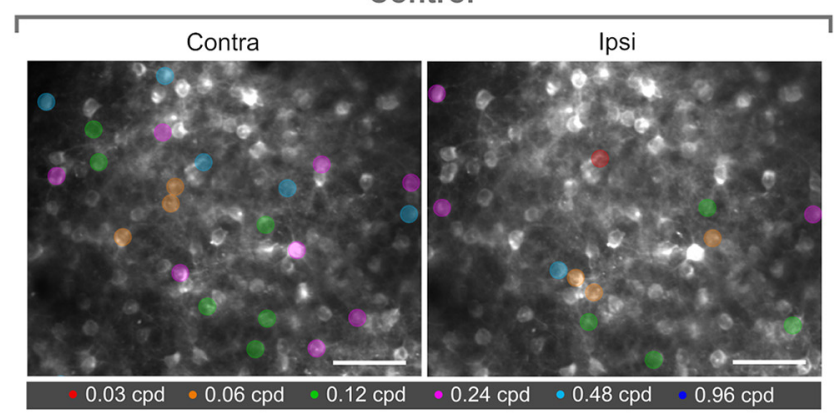

B

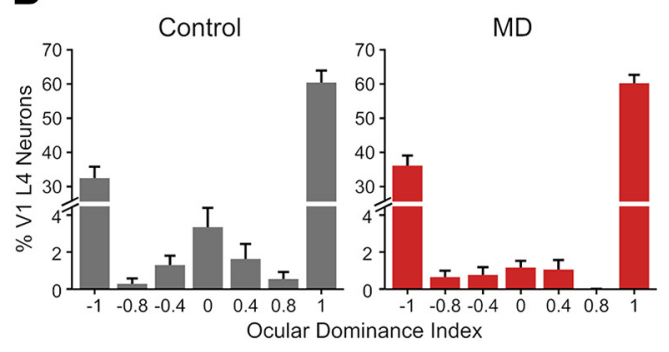

C

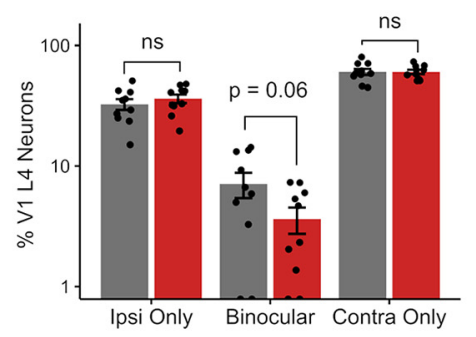

MD

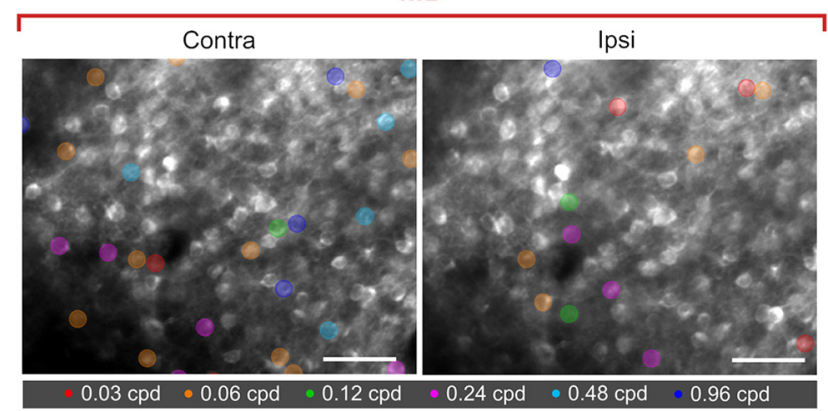

D

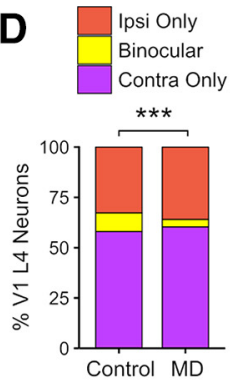

E

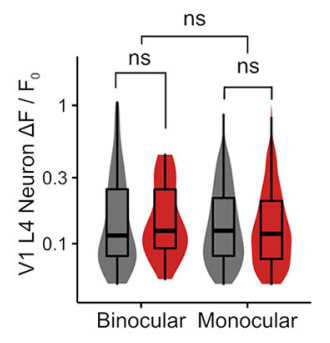

$\mathbf{F}$

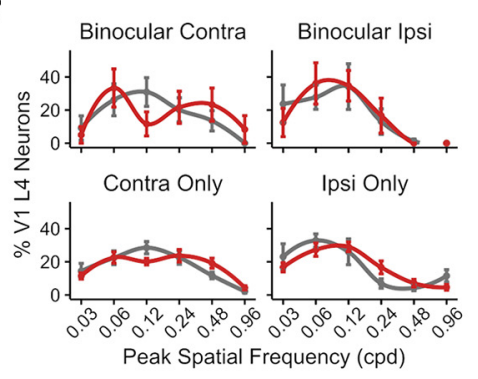

I

$P<0.05$

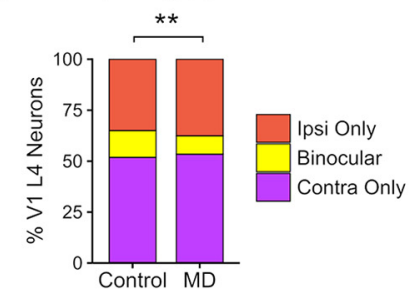

G

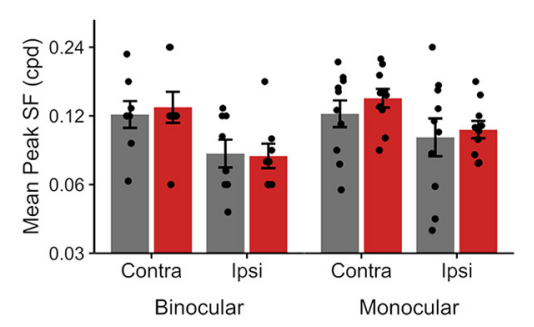

J

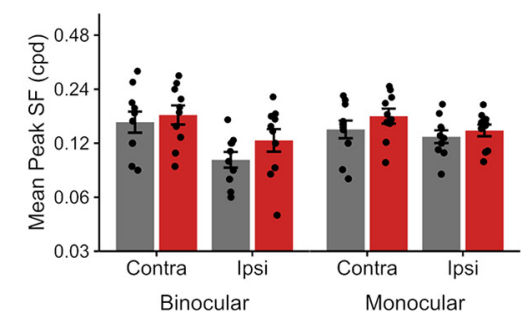

H

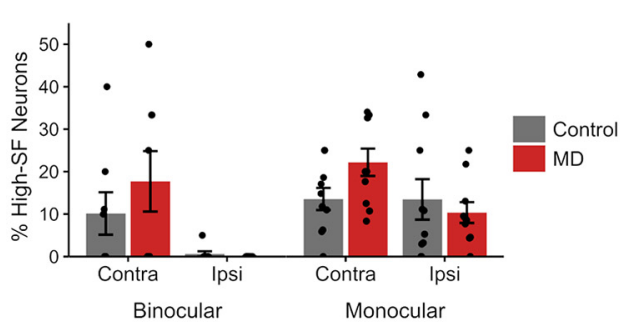

K

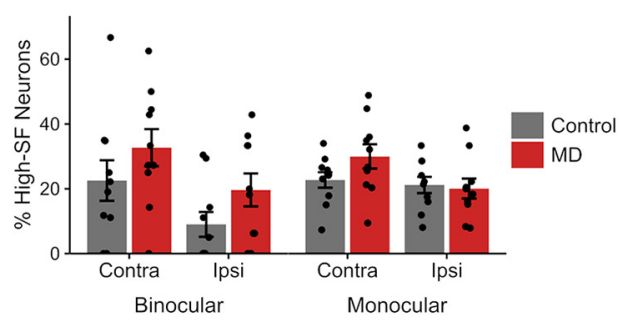

Figure 7. In V1 L4 neurons, long-term critical-period MD leads to reduced binocularity but preserved spatial acuity. A, Example FoVs (summed projection) of bV1 L4 neurons, color-coded according to peak SF of neuron during contralateral-and ipsilateral-eye visual stimulation in control versus MD mice. Scale bar, $100 \mu \mathrm{m}$. B, ODI histogram of V1 L4 neurons in control versus MD mice (mean \pm SEM per field, $n=10$ fields in 3 control mice, 10 fields in 2 MD mice). C, Percentage of visually responsive V1 L4 excitatory neurons that are ipsi-only, binocular, and contra-only per field in control versus MD mice (mean \pm SEM per field). Control, ipsi-only: $32.5 \pm 3.3 \%$; binocular: $7.1 \pm 1.7 \%$; contra-only: $60.4 \pm 3.4 \%$. MD, ipsi-only: $36.1 \pm 2.9 \%$; binocular: $3.6 \pm 0.8 \%$; contra-only: $60.2 \pm 2.4 \%$. Linear mixed-effects model, effect of MD for ipsi-only: $F=0.75, p=0.39 ;$ binocular: $F=3.67, p=0.06$; contra-only: $F=0.002, p=0.96$. $D$, Ipsi-only, binocular, and contra-only fractions of visually responsive V1 L4 neurons in control versus MD mice $\left(\chi_{(2)}^{2}=14.57, p=0.0006\right)$. $\boldsymbol{E}$, Violin and overlaid box plots of response amplitude $R_{\text {pref }}$ of binocular and monocular V1 L4 neurons in control versus MD mice. Linear mixed-effects model, effect of MD: $F=0.08, p=0.78$; binocular versus monocular: $F=2.41, p=0.12$. In box plots, middle mark indicates the median, and bottom and top edges indicate 25th and 75th percentiles, respectively. $F$, Mean probability distribution of peak SF in binocular (top) and monocular (bottom) V1 L4 neurons' contralateral- (left) and ipsilateral-eye (right) responses (mean \pm SEM by field values). Mean values were fitted with a local regression smoothing function. G, Mean peak SF of V1 L4 neurons in control versus MD mice (mean \pm SEM by field values). Linear mixed-effects model, effect of $M D: F=0.70, p=0.53$; binocular versus monocular: $F=2.30, p=0.13$; contra versus ipsi: $F=16.41, p=0.0001$. $\boldsymbol{H}$, Percentage of V1 L 4 neurons with peak SF of $0.48-0.96 \mathrm{cpd}$ (high-SF neurons; mean \pm SEM by field values). Linear mixed-effect model, effect of MD: $F=1.48, p=0.22$; binocular versus monocular: $F=9.19, p=0.003$; contra versus ipsi: $F=14.60, p=0.0002 . \boldsymbol{B}-\boldsymbol{H}, n=572$ neurons in 3 control mice, 565 neurons in $2 \mathrm{MD}$ mice. $\boldsymbol{I}-\boldsymbol{K}$, The decrease in binocular fraction and the lack of acuity deficits following critical-period $\operatorname{MD}(\boldsymbol{D}, \mathbf{G}, \boldsymbol{H})$ are shown using a more liberal statistical criterion $(p<0.05)$ in determining visual responsiveness than the typical criterion used in this study $\left(p<0.01\right.$; see Materials and Methods). I, Same convention as in $\boldsymbol{D}$. $\chi_{(2)}^{2}=9.91, p=0.007 ; 13 \%$ versus $9 \%$ binocular (control vs MD). J, Same convention as $\mathbf{G}$. Linear mixed-effects model, effect of MD: $F=1.42, p=0.31$; binocular versus monocular: $F=1.33, p=0.25$; contra versus ipsi: $F=15.67, p=0.0001$. $\boldsymbol{K}$, Same convention as in $\boldsymbol{H}$. Linear mixed-effect model, effect of $\mathrm{MD}: F=5.43, p=0.02$; binocular versus monocular: $F=0.75, p=0.38$; contra versus ipsi: $F=10.84, p=0.001 . I-K, n=1080$ neurons in 3 control mice, 1163 neurons in $2 \mathrm{MD}$ mice. ns, Not significant at $p>0.05$. ${ }^{* *} p<0.01,{ }^{* *} p<0.001$. 
A

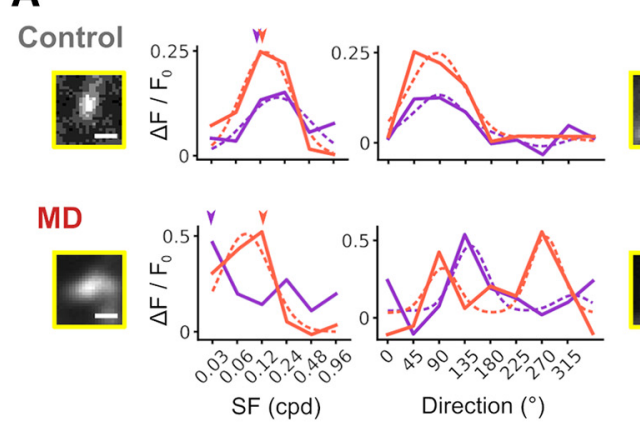

B

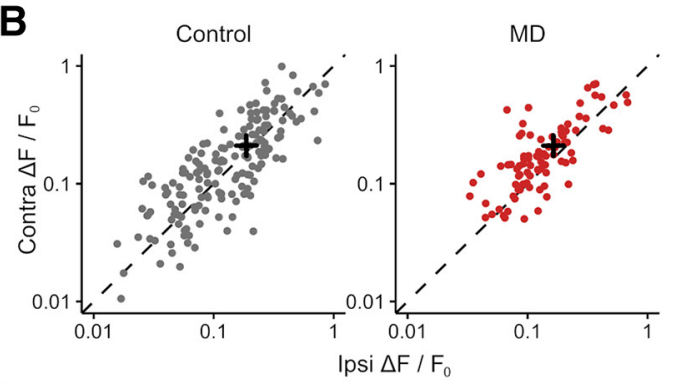

E
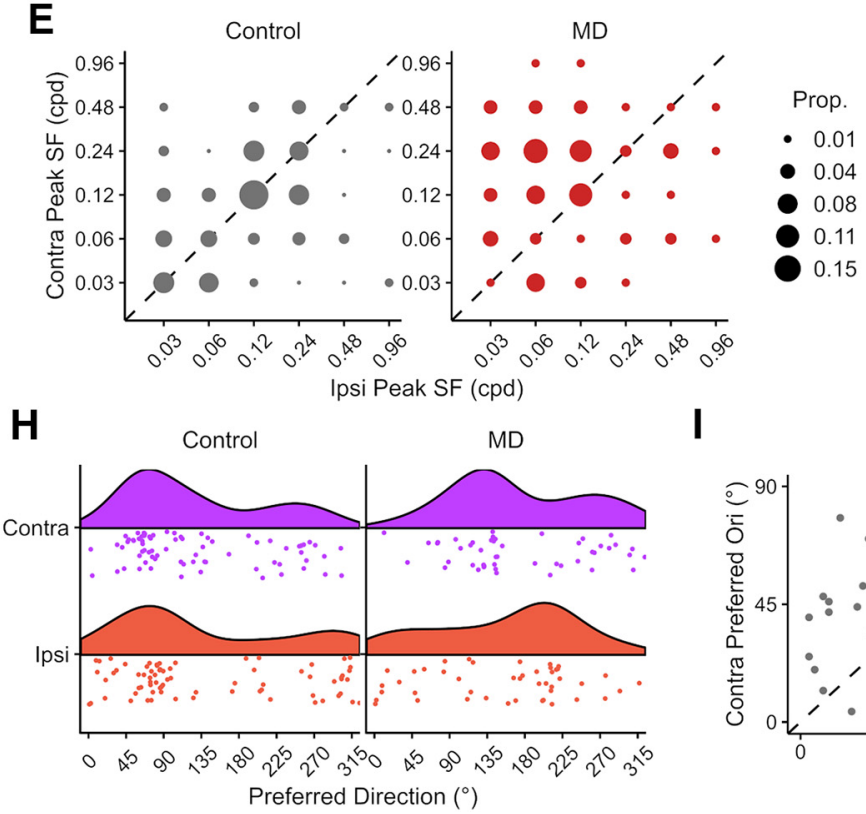
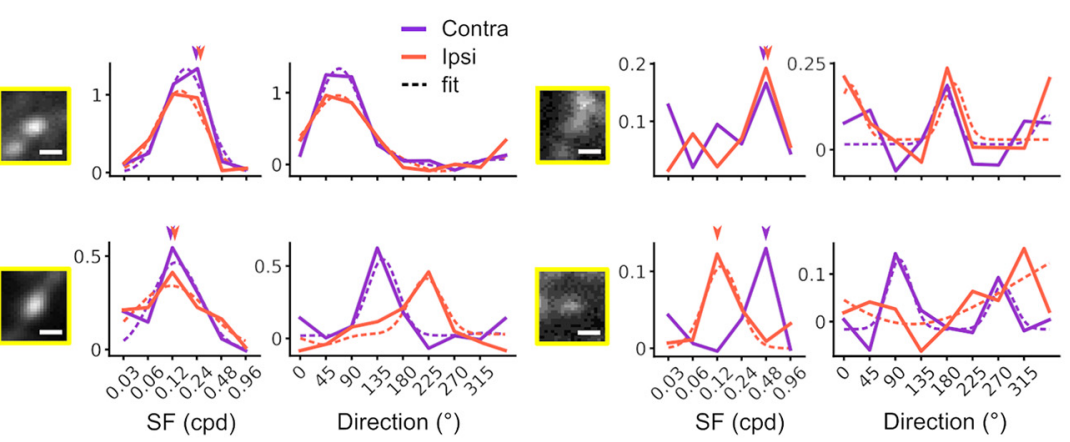

C
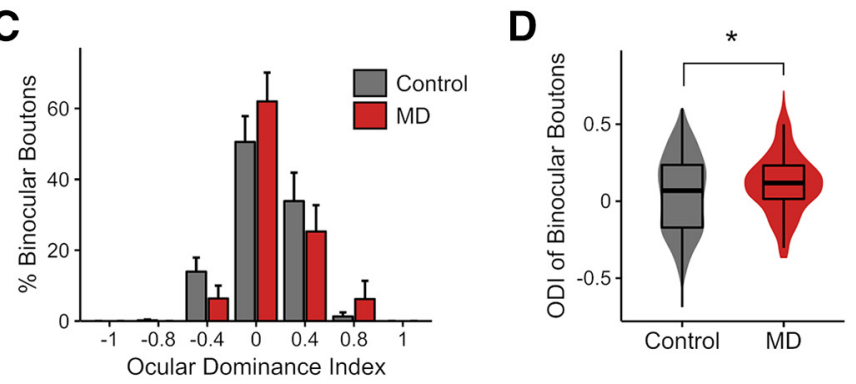
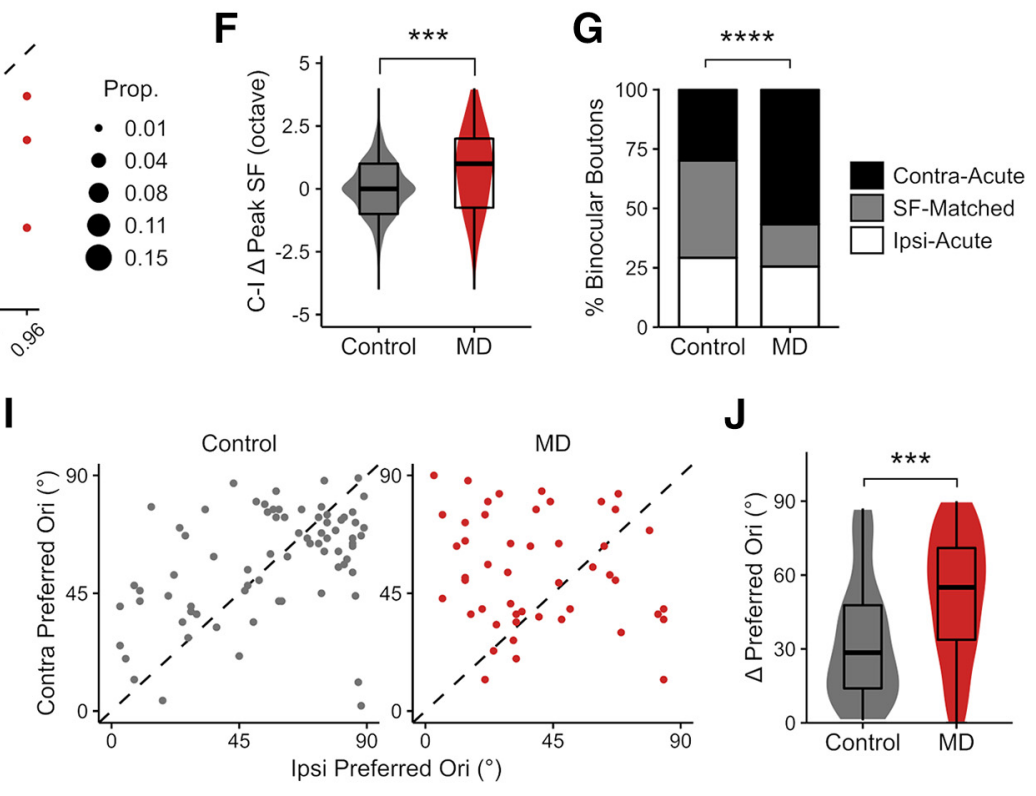

$\mathbf{J}$

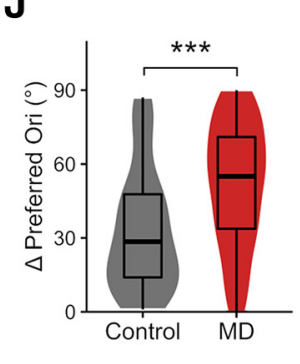

Figure 8. Binocular mismatch in thalamocortical boutons following long-term critical-period MD. A, Example tuning curves of binocular boutons in control (top row) and MD (bottom row) mice (3 examples each). Each pair of plots show SF (left) and orientation tuning (right) at SF indicated with arrowheads of a binocular bouton shown in inset. Scale bar, $2 \mu \mathrm{m}$. Purple represents contralateral-eye trials. Orange represents ipsilateral-eye trials. Solid lines indicate mean response amplitudes. Dotted lines indicate fitted curves based on mean values. Fits omitted if curve-fitting failed to merge. $B$, Scatter plot of response amplitudes of binocular dLGN boutons to preferred stimuli $\left(R_{\text {pref }}\right)$ during contra- ( $y$ axis) versus ipsi-eye $(x$ axis) viewing. Black crosses represent mean values. C, OD distribution of binocular boutons (mean \pm SEM per field, $n=17$ fields in 5 control mice, 20 fields in 6 MD mice). D, Violin and overlaid box plots of ODI values of binocular boutons in control versus MD mice $(t$ test: $p=0.01)$. In box plots, middle mark indicates the median, and bottom and top edges indicate 25 th and 75 th percentiles, respectively. $\boldsymbol{E}$, Proportion plots of contraversus ipsi-eye peak SF of binocular boutons in control versus MD mice. Unity (dotted line) represents perfect match. $\boldsymbol{F}$, Violin and overlaid box plots of interocular difference in peak SF (contra-ipsi, in octaves) for binocular boutons (Wilcoxon rank sum test: $p=0.0008$ ). $\mathbf{G}$, Fractions of SF-matched, Contra-Acute (peak SF is greater in contra-eye response) or Ipsi-Acute (peak SF is greater in ipsi-eye response) binocular boutons in control versus MD mice $\left(\chi_{(2)}^{2}=20.75, p=3 \times 10^{-5}\right)$. $\boldsymbol{H}$, Rain cloud plots represent distributions of preferred direction in orientation- or direction-selective binocular boutons in control versus MD mice. $I$, Scatter plots of preferred orientation of binocular boutons during contra-versus ipsi-eye viewing in control versus $M D$ mice. $J$, Violin and overlaid box plots of interocular difference in preferred orientation for binocular boutons in control versus MD mice (Wilcoxon rank sum test: $p=0.0003$ ). $\boldsymbol{B}-\mathbf{G}, n=171$ binocular boutons in 5 control mice, 90 binocular boutons in $6 \mathrm{MD}$ mice. $\boldsymbol{H}-\boldsymbol{J}, n=74$ (control) and 48 (MD) OS/DS binocular boutons. ${ }^{*} p<0.05$, ${ }^{* * *} p<0.001,{ }^{* * * *} p<0.0001$.

whether dLGN binocular modulation is affected by long-term criticalperiod MD by examining dLGN boutons' activity during monocular and binocular viewing conditions in control and MD mice.

We first categorized binocular modulation of dLGN boutons into three broad modes: binocular activation ("both only"), binocular "suppression," and "remaining responsive" (Fig. 10A).
"Both only" boutons displayed significant visual responses only during binocular viewing but not during any of the monocular viewing conditions and accounted for $\sim 30 \%$ of dLGN boutons in controls (Fig. 10B-E). Binocularly "suppressed" boutons were visually responsive during one (or both) of monocular viewing conditions but not during binocular viewing and represented 
A

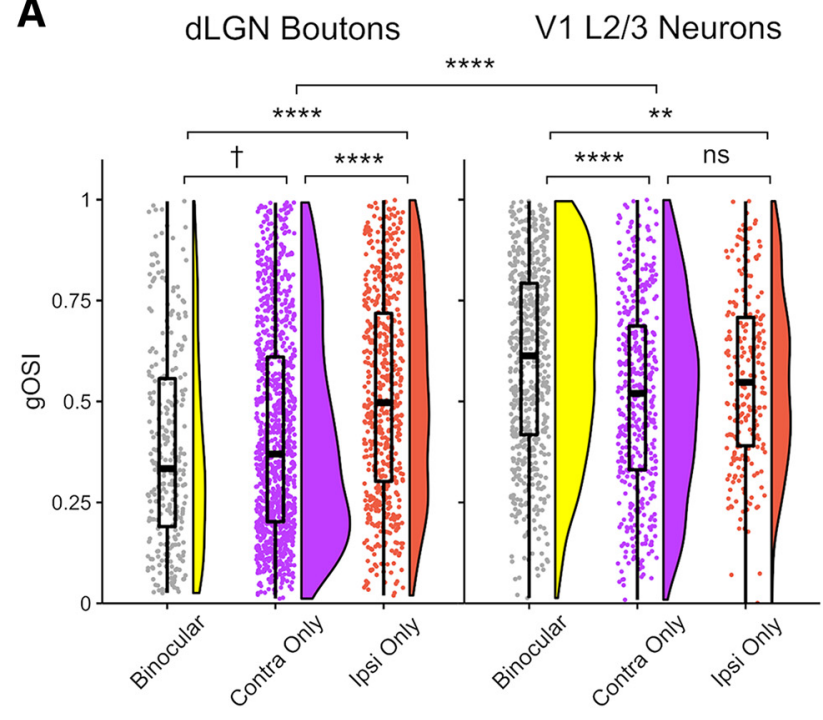

C

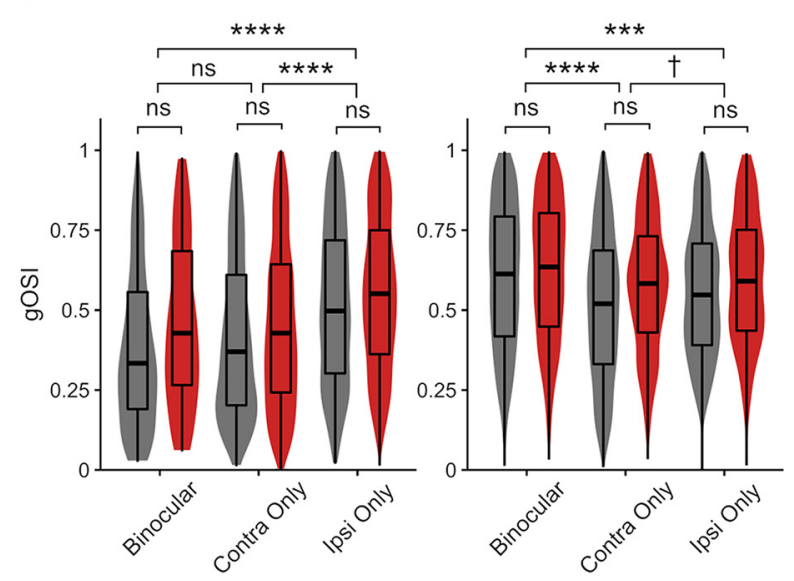

B

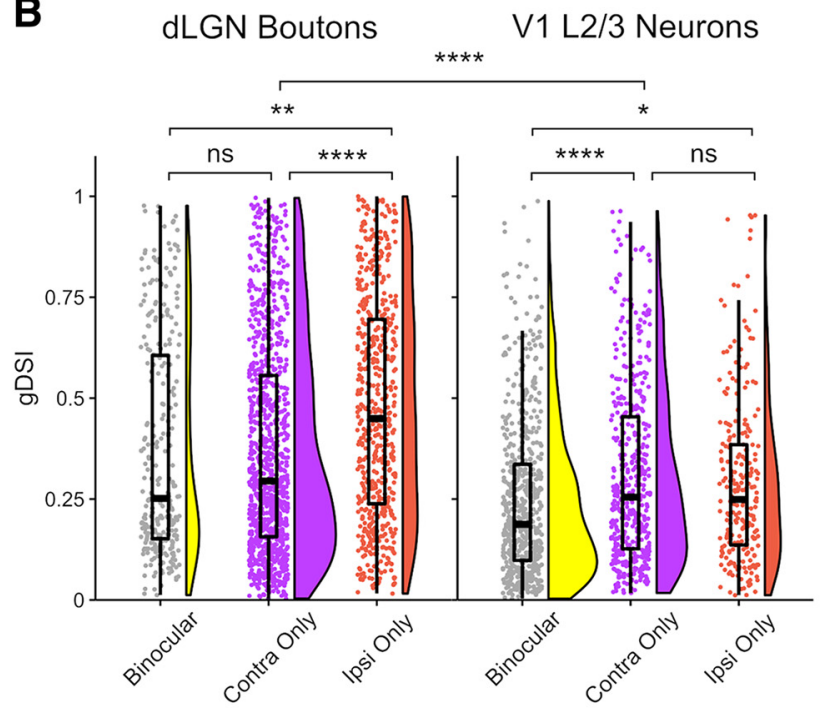

D dLGN Boutons

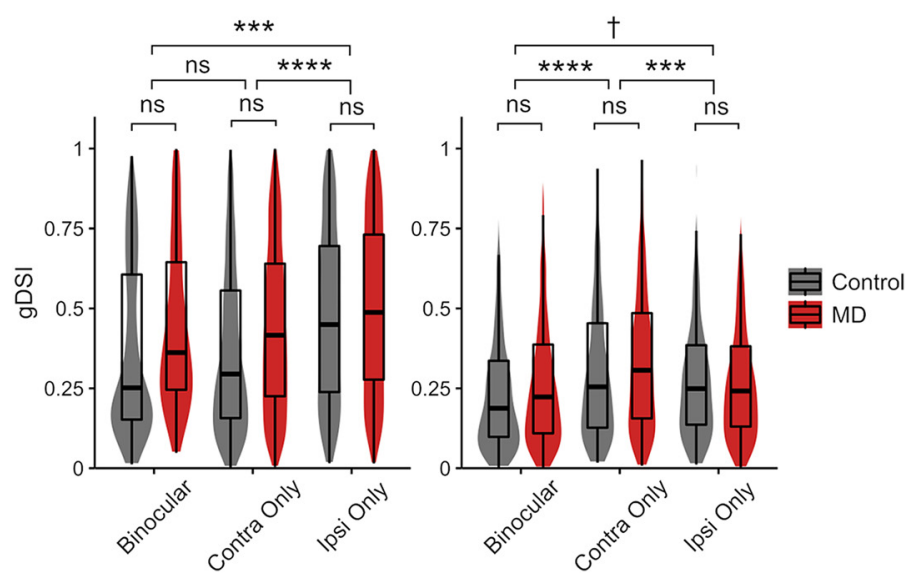

Figure 9. Comparison of orientation/direction tuning properties of $\mathrm{dLGN}$ boutons versus V1 L2/3 neurons. $\boldsymbol{A}, \boldsymbol{B}$, Control data only. $\boldsymbol{A}$, Raincloud plots represent distributions of global orientation selectivity index (gOSI) values in binocular, contra-only, and ipsi-only boutons (left) and V1 L2/3 neurons (right). V1 L2/3 neurons are more orientation-selective than dLGN boutons. Linear mixed-effects models. Boutons versus $\mathrm{L} 2 / 3$ neurons: $F=10.85, p=2 \times 10^{-5}$; boutons: binocular versus contra-only: $F=2.98, p=0.08$; binocular versus ipsi-only: $F=44.28, p=7 \times 10^{-11}$; contra-only versus ipsi-only: $F=15.25, p=9 \times 10^{-5}$. V1 L2/3 neurons: binocular versus contra-only: $F=23.98, p=1 \times 10^{-6} ;$ binocular versus ipsi-only: $F=9.91, p=0.001$; contra-only versus ipsi-only: $F=1.41, p=0.23$. $B$, Distributions of global direction selectivity index ( $g D S I$ ) values in binocular, contra-only, and ipsi-only boutons and V1 L2/3 neurons (same convention as $A$ ). dLGN boutons are more direction-selective than V1 L2/3 neurons. Linear mixed-effects models: Boutons versus L2/3 neurons: $F=$ $15.90, p=1 \times 10^{-7}$; boutons: binocular versus contra-only: $F=0.12, p=0.72$; binocular versus ipsi-only: $F=8.23, p=0.004 ;$ contra-only versus ipsi-only: $F=34.46, p=5 \times$ $10^{-9}$. V1 L2/3 neurons: binocular versus contra-only: $F=23.53, p=1 \times 10^{-6}$; binocular versus ipsi-only: $F=6.42, p=0.01 ;$ contra-only versus ipsi-only: $F=1.31, p=0.25$. $C$, Violin and overlaid box plots of gOSI in dLGN boutons (left) and V1 neurons (right) in control versus MD mice. Linear mixed-effects models. Boutons: effect of MD: $F=1.01, p=0.33$; binocular versus contra-only: $F=1.96, p=0.16$; binocular versus ipsi-only: $F=41.71, p=1 \times 10^{-10}$; contra-only versus ipsi-only: $F=43.59, p=4 \times 10^{-11}$. V1 L2/3 neurons: effect of MD: $F=0.95, p=0.34$; binocular versus contra-only: $F=32.54, p=1 \times 10^{-8}$; binocular versus ipsi-only: $F=11.44, p=0.0007$; contra-only versus ipsi-only: $F=2.81$, $p=0.09$. D, Violin and overlaid box plots of gDSI in dLGN boutons (left) and V1 neurons (right) in control versus MD mice. Linear mixed-effects models. Boutons: effect of MD: $F=1.37$, $p=0.26$; binocular versus contra-only: $F=0.0009, p=0.97$; binocular versus ipsi-only: $F=11.38, p=0.0007$; contra-only versus ipsi-only: $F=44.76, p=2 \times 10^{-11}$. V1 L2/3 neurons: effect of MD: $F=1.03, p=0.32$; binocular versus contra-only: $F=50.41, p=1 \times 10^{-12}$; binocular versus ipsi-only: $F=3.38, p=0.06$; contra-only versus ipsi-only: $F=$ $14.25, p=0.0001$. In box plots, middle mark indicates the median, and bottom and top edges indicate 25 th and 75 th percentiles, respectively. All panels: $n=2866$ boutons in 5 control mice, 3503 boutons in 6 MD mice; $1051 \mathrm{~V} 1 \mathrm{~L} 2 / 3$ neurons in 9 control mice, $1355 \mathrm{~L} 2 / 3$ neurons in 6 MD mice. ns, Not significant at $p>0.1 .{ }^{\dagger} p<0.1,{ }^{*} p<0.05,{ }^{* *} p<0.01,{ }^{* * *} p<$ $0.001,{ }^{* * * *} p<0.0001$.

$\sim 50 \%$ of dLGN boutons in controls (Fig. $10 \mathrm{D}, \mathrm{E}$ ). The remaining boutons, those that were visually responsive during monocular and binocular viewing, were categorized as "remaining responsive," and they made up $~ 20 \%$ of dLGN boutons in controls (Fig. $10 D, E)$. "Both only" boutons were surprisingly numerous, similar in proportion to ipsi-only monocular boutons (Fig. 10B-G). "Both only" boutons displayed generally smaller visual responses compared with other boutons (Fig. $10 H$ ), suggesting that their activity is probably too low to be detected during monocular viewing conditions.
We found that long-term critical-period MD leads to a significant reduction in the percentage of dLGN boutons "remaining responsive" during binocular viewing (Fig. 10D,E). Among eye groups, including "both only," the percentage of binocular dLGN boutons was significantly lower in MD mice compared with controls (Fig. 10F,G), consistent with our observation from monocular viewing conditions (Fig. 1I). To examine binocular modulation of response strength, we considered responses from all visually responsive dLGN boutons during each viewing con- 
A

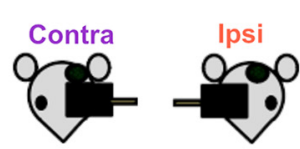

Eye Group

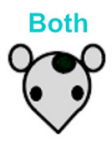

Bin. Modulation

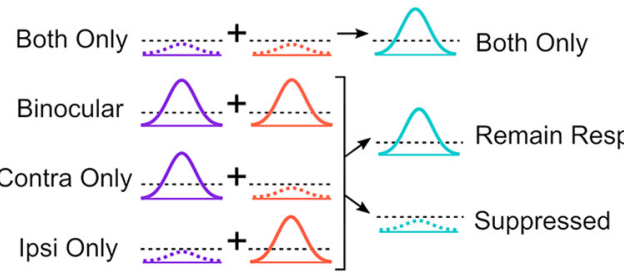

B

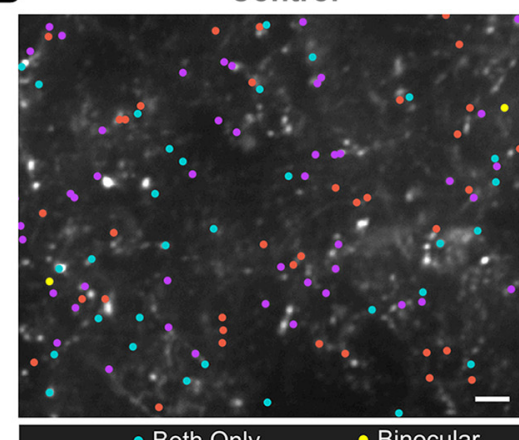

MD

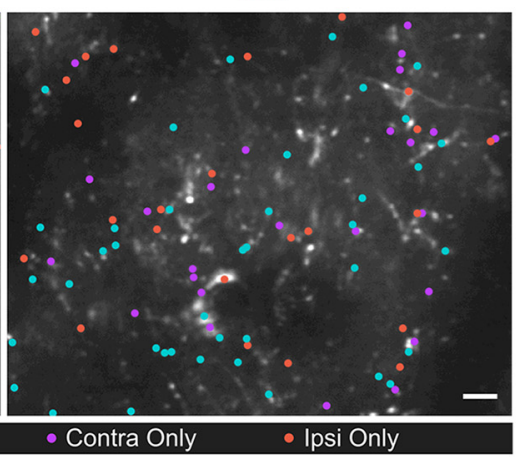

C
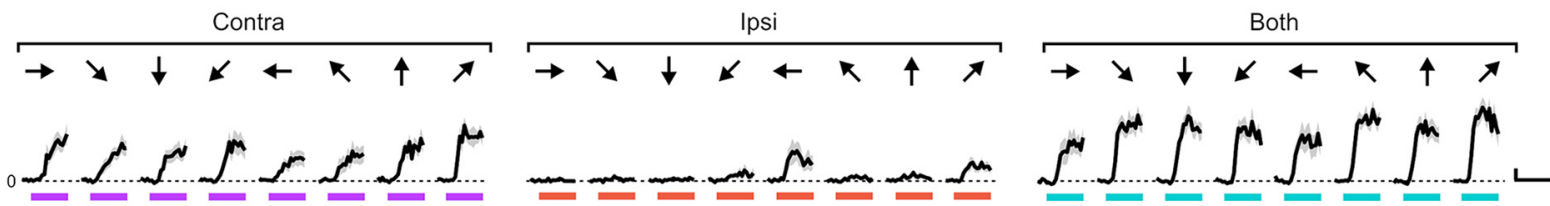

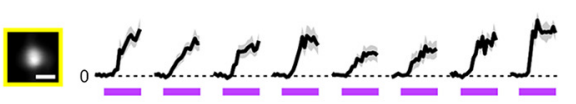

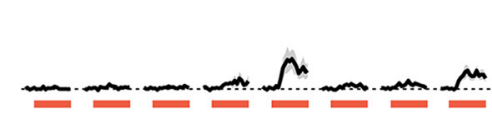

Q
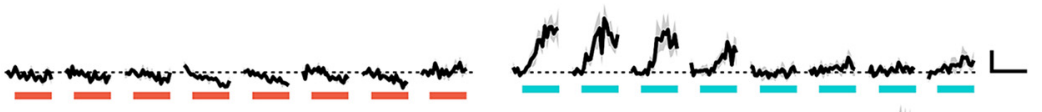

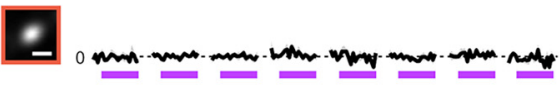

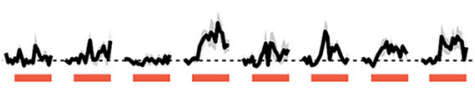

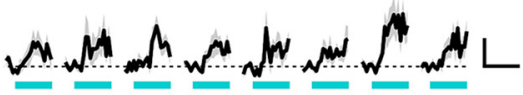

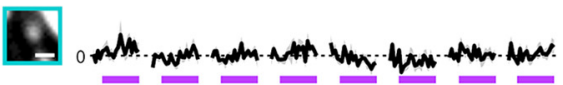
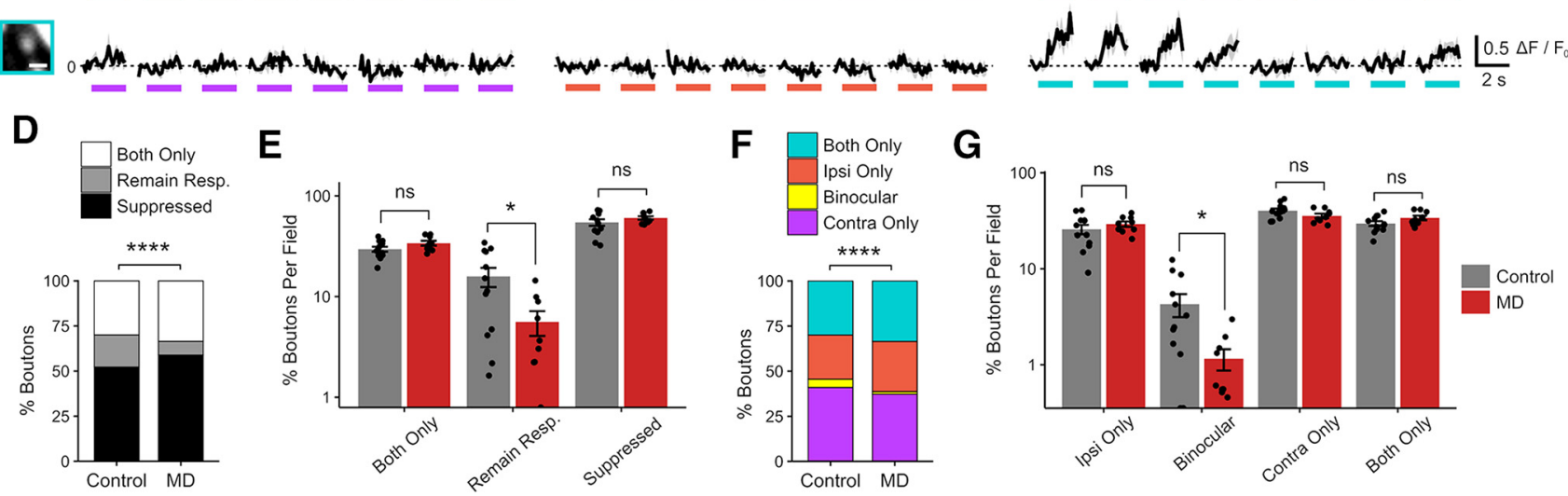

E

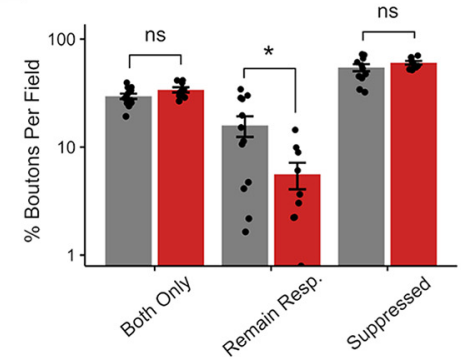

H

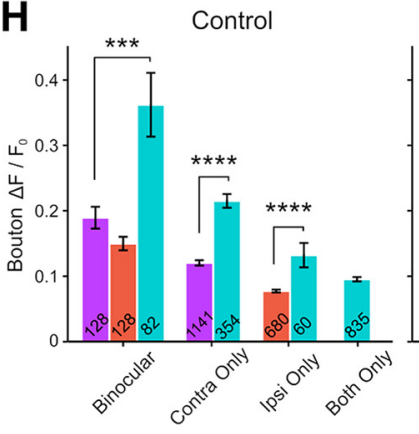

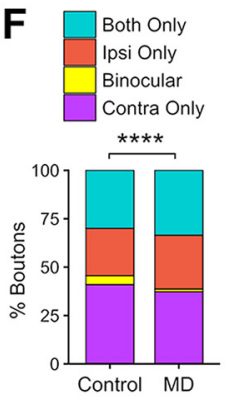

G

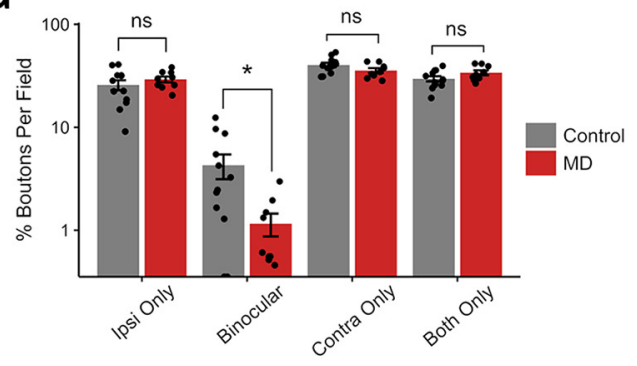

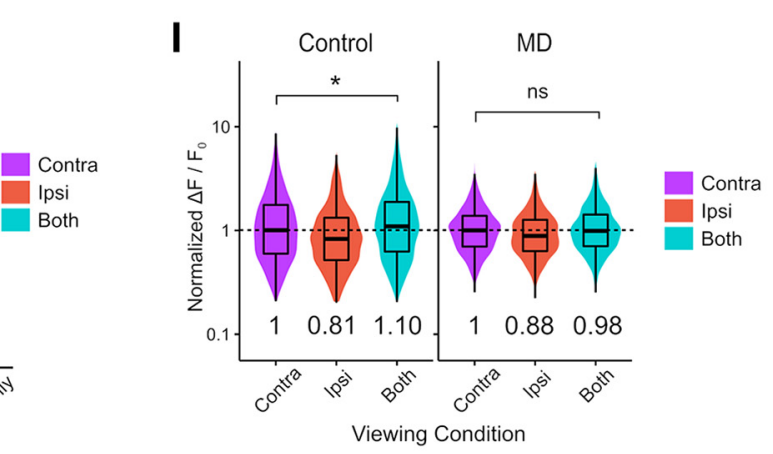

Figure 10. Long-term critical-period MD impairs binocular facilitation of thalamocortical boutons. $\boldsymbol{A}$, Illustration of three modes of binocular modulation of visual responses: both only, remaining responsive, and suppressed. Broken lines indicate noise floor. $\boldsymbol{B}$, Example FOVs (summed projection) of dLGN boutons imaged in bV1, color-coded according to eye group, including both-only in control versus MD mice. Scale bar, $10 \mu \mathrm{m}$. C, Example Ca ${ }^{2+}$ signals in a binocular (top), contra-only and ipsi-only (middle), and both-only (bottom) boutons in response to drifting gratings presented to contra-eye, ipsi-eye, and both eyes. Black represents mean trace. Gray represents mean \pm SEM of 8 repeats. Colored bars represent time of stimulus presentation. Scale bar, $2 \mu \mathrm{m}$. Responses to 8 orientations at peak SF are shown. $D$, Fractions of dLGN boutons according to mode of binocular modulation in control versus MD mice $\left(\chi_{(2)}^{2}=119.40, p=2 \times 10^{-16}\right)$. $\boldsymbol{E}$, Percentage of boutons that are both-only, remaining responsive, suppressed per field in control versus MD mice (mean \pm SEM per field). Control, both-only: $29.6 \pm 1.6 \%$; remaining responsive: $15.8 \pm 3.4 \%$; suppressed: $54.5 \pm 4.1 \%$. MD, both-only: $33.9 \pm 1.8 \%$; remaining responsive: $5.6 \pm 1.5 \%$; suppressed: $60.4 \pm 2.3 \%$. $t$ tests, effect of MD for both-only: $p=0.10$; remaining responsive: $p=0.01$; suppressed: $p=0.22 ; n=12$ fields in 5 control mice, 9 fields in 5 MD mice. $F$, Fractions of visually responsive dLGN boutons according to eye group in control versus MD mice $\left(\chi_{(3)}^{2}=57.23, p=2 \times 10^{-12}\right)$.G, Percentages of boutons in each eye group (ipsi-only, binocular, and contra-only, both-only) in control versus MD mice (mean \pm SEM per field). $t$ tests, effect of MD for ipsi-only: $p=0.32$; binocular: $p=0.02$; contra-only: $p=0.10$; both-only: $p=0.10$. $\boldsymbol{H}$, Response amplitude $\mathrm{R}_{\text {pref }}$ of boutons under different viewing conditions (mean \pm SEM of all sample). Linear mixed-effects models. Control: contra-eye versus both-eye viewing in binocular boutons: $F=17.07, p=5 \times 10^{-5}$; monocular versus both-eye viewing in contra-only boutons: $F=79.16$, $p=2 \times 10^{-16}$; in ipsi-only boutons: $F=53.39, p=7 \times 10^{-13} ;$ MD: contra-eye versus both-eye viewing in binocular boutons: $F=0.003$, (Figure legend continues.) 

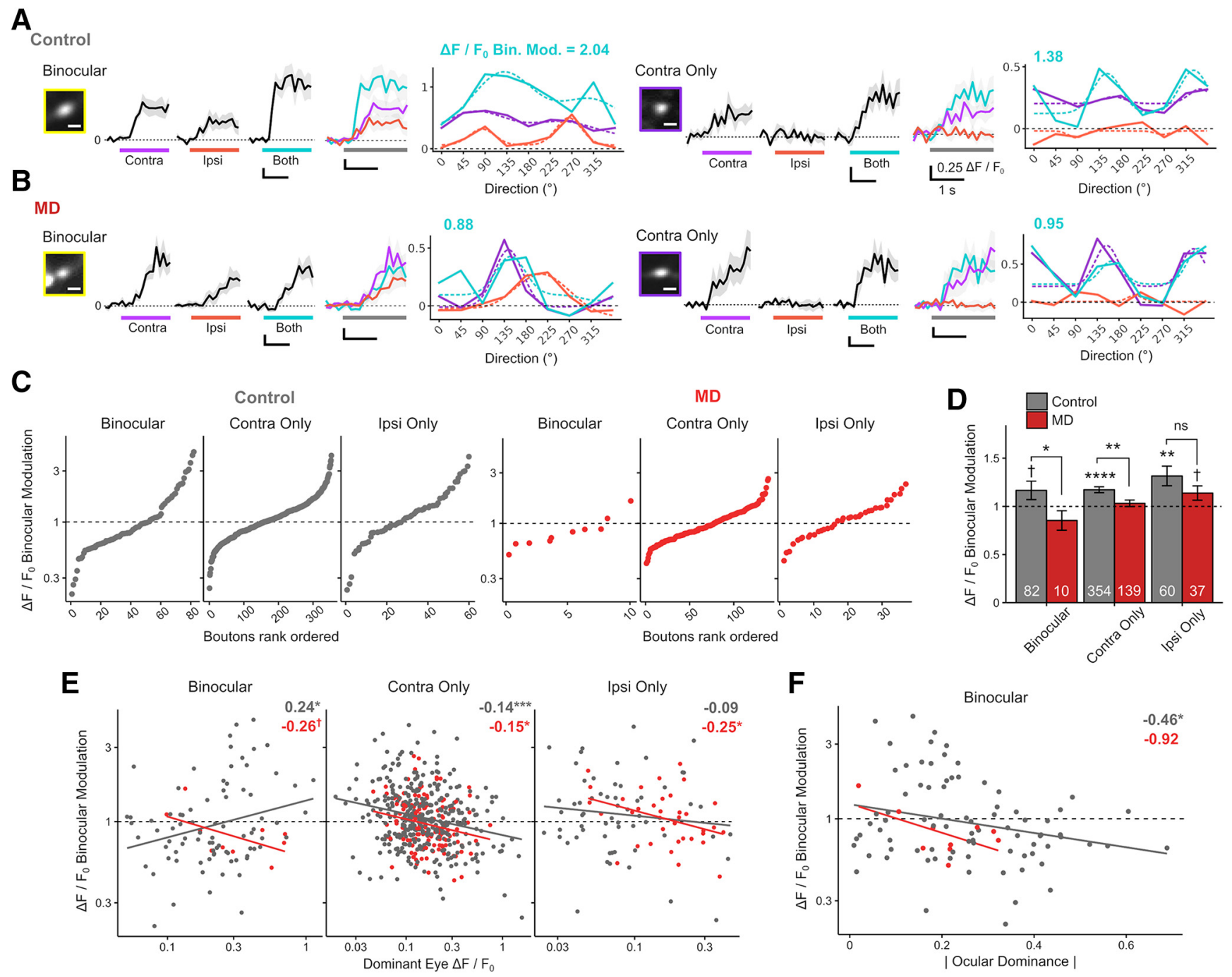

MD
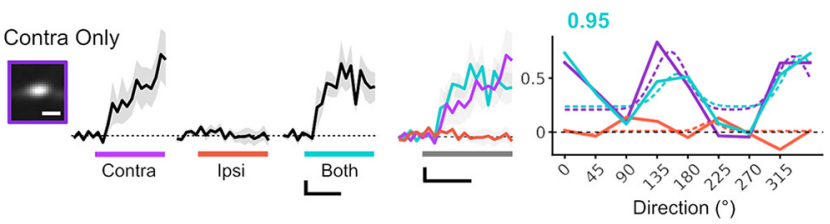

C
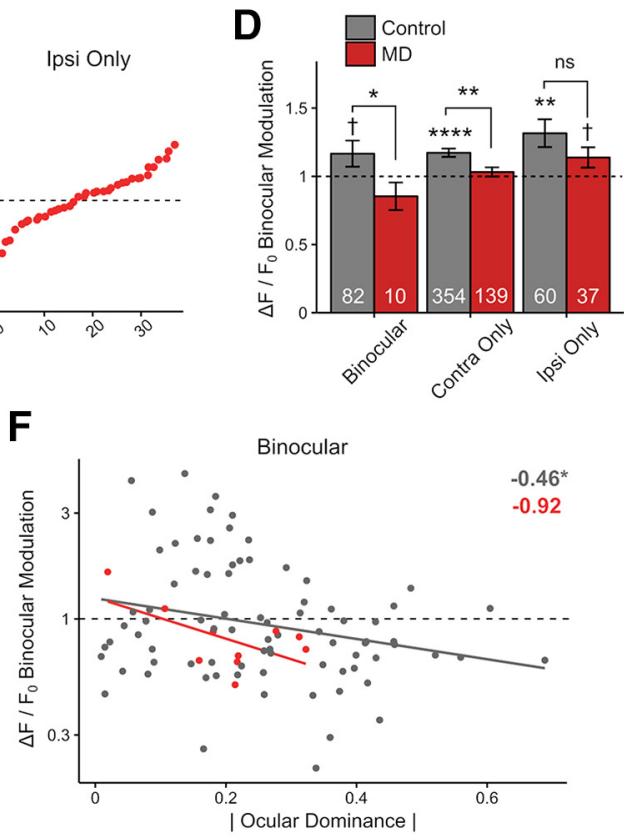

Figure 11. Long-term MD-induced impairment of binocular modulation affects binocular and monocular thalamocortical boutons. $A, B$, Normalized $\mathrm{Ca}^{2+}$ signals from two example dLGN boutons from control $(\boldsymbol{A})$ and MD $(\boldsymbol{B})$ mice. Binocular dLGN boutons (left) and contra-only monocular boutons (right), as well as their respective bouton images and orientation tuning curves. Traces are in response to drifting gratings presented to contra-eye, ipsi-eye, and both eyes. Black represents mean trace. Gray represents mean \pm SEM of 8 repeats. Colored bars represent time of stimulus

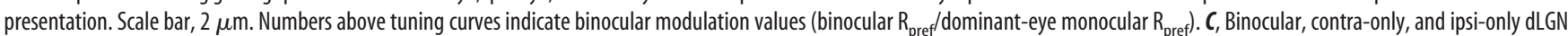
boutons are shown rank-ordered according to binocular modulation. Both binocular facilitation and suppression exist in control (left) and MD (right) mice. $D$, Binocular modulation of visual responses for binocular, contra-only, and ipsi-only boutons in control versus MD mice (mean \pm SEM of all sample; numbers inside bars indicate number of boutons; $t$ tests against 1 for binocular control: $p=0.08$; contra-only control: $p=2 \times 10^{-8}$; ipsi-only control: $p=0.002$; binocular MD: $p=0.18$; contra-only MD: $p=0.34$; ipsi-only MD: $p=0.07$.t tests between control versus MD for binocular: $p=0.03$; contra-only: $p=0.002$; ipsi-only: $p=0.16$. E, Scatter plots and linear regression of binocular modulation as a function of dominant-eye monocular $\mathrm{R}_{\text {pref }}$ for binocular, contra-only, and ipsi-only boutons in control (gray) and MD (red) mice. $\boldsymbol{F}$, Scatter plots and linear regression of binocular modulation as a function of absolute $0 D$ for binocular boutons in control (gray) and MD (red) mice. $\mathbf{C}-\boldsymbol{F}$, Broken lines indicate no binocular modulation. $\boldsymbol{E}, \boldsymbol{F}$, Numbers on top right indicate estimated slope (symbols indicate $p$ values). Solid colored lines indicate linear regression fits. Gray represents control. Red represents MD. $\mathbf{C}-\boldsymbol{F}, n=496$ boutons in 5 control mice, 186 boutons in 4 MD mice. ns, Not significant at $p>0.1 .{ }^{\dagger} p<0.1,{ }^{*} p<0.05,{ }^{* *} p<0.01$, ${ }^{* * *} p<0.001,{ }^{* * * *} p<0.0001$.$$
\leftarrow
$$

(Figure legend continued.) $\quad p=0.96$; monocular versus both-eye viewing in contra-only boutons: $F=77.93, p=2 \times 10^{-16}$; in ipsi-only boutons: $F=75.43, p=2 \times 10^{-16}$. Numbers inside bars indicate numbers of boutons. $I$, Violin and overlaid box plots of response amplitude $R_{\text {pref }}$ of boutons during contra-eye, ipsi-eye, and both-eye viewing conditions, normalized to contra-eye viewing median values (medians indicated by numbers below violin plots). Linear mixed-effects models, contra-eye versus both-eye viewing in controls: $F=4.59$, $p=0.03$; in MD mice: $F=1.06, p=0.30$. In box plots, middle mark indicates the median, and bottom and top edges indicate 25 th and 75 th percentiles, respectively. $D-I, n=2784$ boutons in 5 control mice, 2443 boutons in 5 MD mice. ns, Not significant at $p>0.05 .{ }^{*} p<0.05$, ${ }^{* * *} p<0.001,{ }^{* * * *} p<0.0001$.
}

dition (contra-eye, ipsi-eye, or both-eye viewing) and normalized them to the median value during contra-eye viewing. We found that, in controls, dLGN boutons displayed overall larger visual responses during both-eye viewing compared with contra-eye viewing (Fig. 10I). However, such binocular facilitation was not observed in MD mice (Fig. 10I). These findings indicate that long-term critical-period MD leads to fewer dLGN boutons remaining responsive during binocular viewing, less binocular boutons, and reduced facilitation of visual responses during both-eye viewing.

Long-term MD-induced impairment of binocular modulation affects both binocular and monocular thalamocortical boutons We next investigated how long-term critical-period MD affects binocular modulation at the level of individual dLGN boutons, 
A

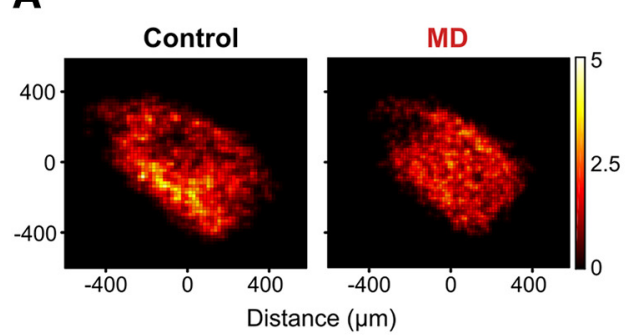

C

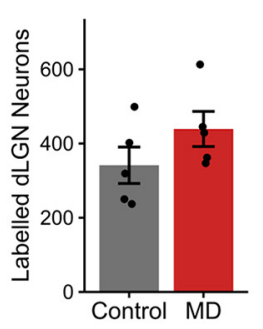

B

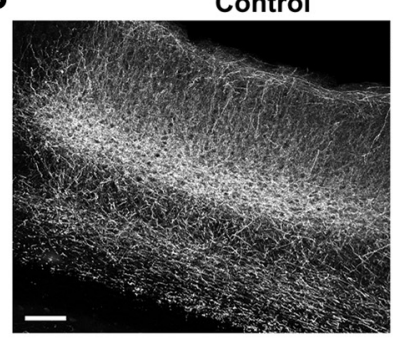

E

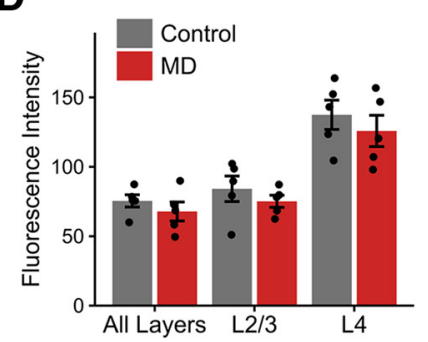

Control
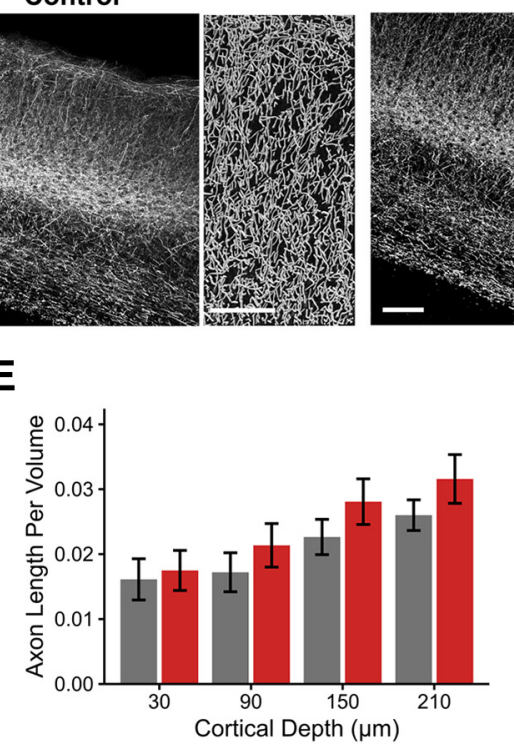

MD

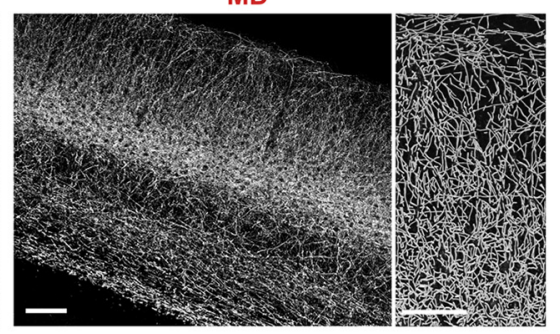

$\mathbf{F}$

Figure 12. No evident structural loss of thalamocortical connectivity following long-term critical-period MD. $A$, Heatmaps showing spatial distribution of dLGN neurons labeled following GCaMP6s virus injection in control (left) and MD (right) mice. Of 10 mice included in this dataset, 6 were part of functional dataset obtained using in vivo two-photon calcium imaging. Heatmaps are based on summed cell counts across all sections and mice. $\boldsymbol{B}$, Left, Example fluorescence images (maximal projection of confocal z stacks) of V1 coronal sections showing dLGN axon labeling in control and MD mice. Scale bar, $100 \mu \mathrm{m}$. Sections were immunostained for GFP. Right, Semiautomatically traced axons in L1-2/3 in control and MD mice. Scale bar, $50 \mu \mathrm{m}$. Images in $\boldsymbol{B}$ are from the same mice shown in Figure 3A. C, Number of dLGN neurons labeled in control versus MD mice (mean \pm SEM by animal; $t$ test: $p=0.19$ ). $\boldsymbol{D}$, Mean fluorescence intensity of labeling in V1 sections from control versus MD mice, shown for all layers, L2/3 only, and L4 only (mean \pm SEM by animal; two-way ANOVA, effect of MD: $F=1.95, p=0.18$, effect of layer: $F=31.14, p=2 \times 10^{-7}$ ). $E$, Traced axon length per volume $\left(\mu \mathrm{m}\right.$ per $\mu \mathrm{m}^{3}$ ) across binned cortical depths in V1 L1-2/3 in control versus MD mice (mean \pm SEM by section; linear mixed-effects model, effect of MD: $F=0.04, p=0.84$, effect of cortical depth: $F=65.64, p=8 \times 10^{-13}$ ). $F$, Violin and overlaid box plots represent distribution of traced axon radius in V1 L1-2/3 in control versus MD mice (linear mixed-effects model, effect of MD: $F=0.35, p=0.57$ ). In box plots, middle mark indicates the median, and bottom and top edges indicate 25 th and 75 th percentiles, respectively. All panels, $n=5$ control and $5 \mathrm{MD}$ mice, 3 sections per animal.

focusing on boutons that remained responsive during binocular viewing. We expressed binocular modulation as a ratio by dividing each dLGN bouton's both-eye $\mathrm{R}_{\text {pref }}$ amplitude by its dominant-eye monocular $\mathrm{R}_{\text {pref }}$ amplitude. Binocular modulation of 1 indicates no change during binocular viewing. We observed both binocular facilitation (binocular modulation $>1$ ) and suppression (binocular modulation $<1$ ) in control and MD mice (Fig. $11 A-C$ ). In control mice, the mean binocular modulation was significantly greater than 1 for contra-only and ipsionly monocular dLGN boutons and showed a trend of being $>1$ for binocular boutons (Fig. 11D). The mean binocular modulation $( \pm$ SEM) was $1.17 \pm 0.09$ for binocular, $1.17 \pm 0.03$ for contra-only, and $1.31 \pm 0.10$ for ipsi-only dLGN boutons. In controls, the mean binocular modulation for all dLGN boutons was 1.19 , indicating that visual responses were enhanced by a factor of 1.19 during binocular viewing compared with monocular viewing.

We found that long-term critical-period MD leads to reduced binocular facilitation of dLGN boutons. Overall, a smaller percentage of dLGN boutons displayed binocular facilitation in MD mice compared with controls (MD: 45\%; control: 53\%). Moreover, the mean binocular modulation was significantly lower in MD mice compared with controls for binocular and contra-eye dominated monocular boutons (Fig. 11D). The mean binocular modulation of all boutons ( \pm SEM) was significantly lower in MD mice compared with controls (MD: $1.04 \pm 0.02$; control: $1.19 \pm 0.02$; $t$ test: $p=0.00058$ ).

Since some dLGN boutons are facilitated while others are suppressed during binocular viewing, we explored factors that might contribute to binocular modulation. We found that, for monocular dLGN boutons, there was a negative correlation between dominant-eye monocular-viewing response strength and binocular modulation (Fig. 11E, middle, right), such that dLGN boutons with stronger monocular responses through the dominanteye tended to show binocular suppression, whereas those with weaker monocular responses tended to be binocularly facilitated. Binocular dLGN boutons in controls, however, displayed an opposite relationship, with stronger boutons showing more binocular facilitation compared with weaker boutons (Fig. 11E, left). Furthermore, binocular dLGN boutons in controls exhibited a significant correlation between the degree of binocularity and binocular modulation (Fig. $11 F$ ); boutons that were more binocular, more closely matched in response amplitude between the eyes, displayed greater degrees of binocular facilitation than those that were less well matched. This correlation between binocularity and binocular modulation among binocular dLGN boutons was not significant in MD mice. These data demonstrate that long-term critical-period MD leads to an impairment of binocular modulation at the level of individual dLGN inputs, impacting both binocular and monocular dLGN inputs.

\section{No evident structural loss of thalamocortical connectivity following long-term critical-period MD}

Finally, we examined whether long-term critical-period MD leads to a structural loss of thalamocortical axons from dLGN to V1. Considering that binocular dLGN inputs constitute a relatively small proportion of the total thalamocortical input and their function is particularly vulnerable to critical-period MD (Figs. 1, 2, 8), we hypothesized that there would be little to no structural deficit in thalamocortical projections following MD. Indeed, we found no significant long-lasting alterations in the density and thickness of dLGN axons in V1 L1-2/3 following 


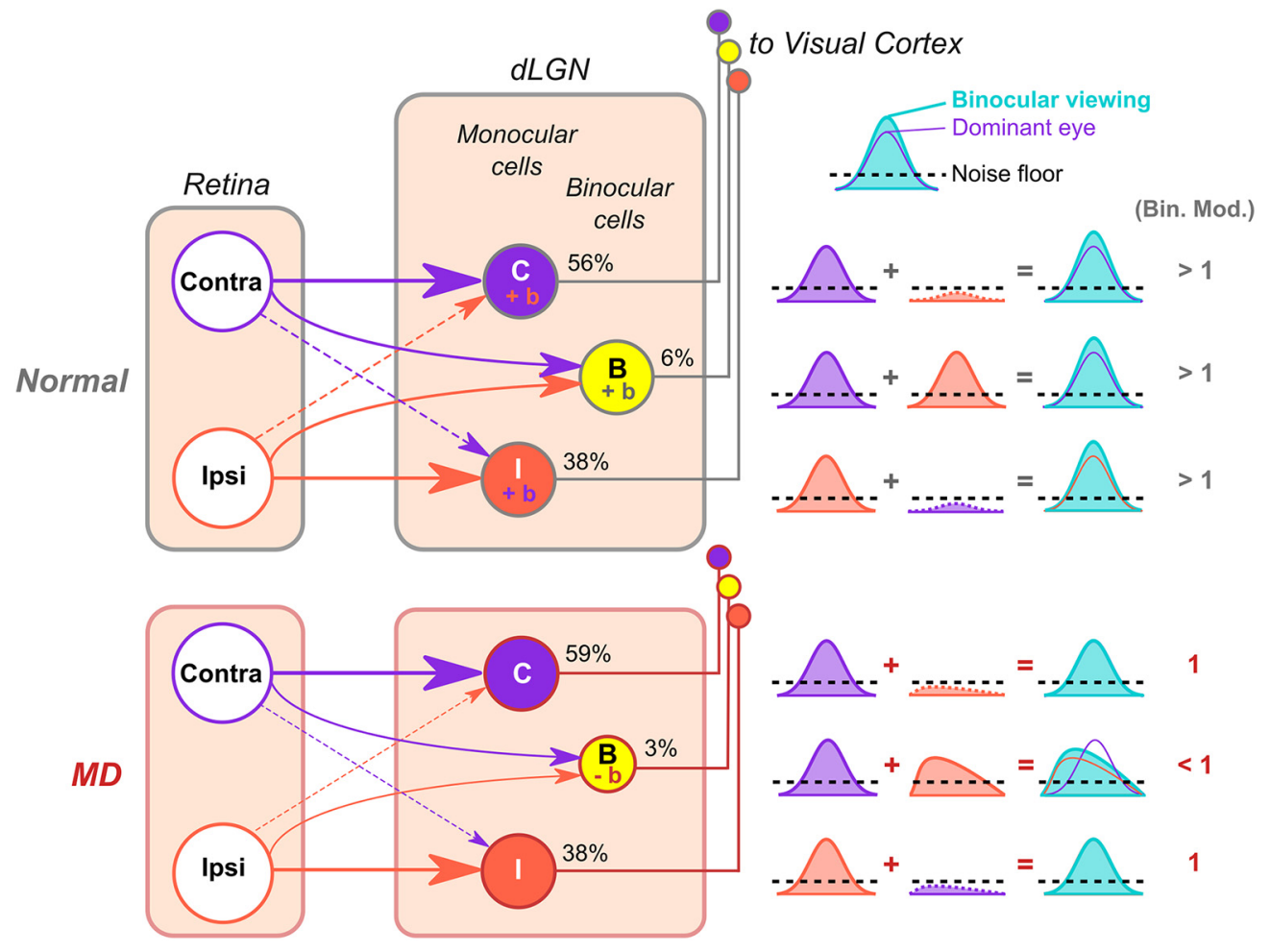

Figure 13. Schematic model of abnormal binocular integration in mouse dLGN following long-term critical-period MD. Summary of main findings. In normal mice, binocular dLGN neurons relay

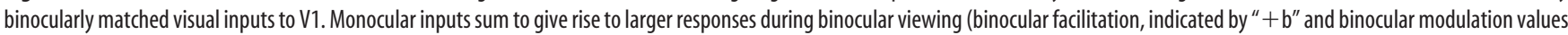
of $>1$ ). Monocular dLGN neurons also display binocular facilitation because the input from the nondominant eye, albeit subthreshold, acts in synergy with the dominant-eye input. In MD mice, the percentage of binocular dLGN neurons is reduced, and surviving binocular neurons relay mismatched visual information to V1. MD mice lack binocular facilitation of binocular and monocular dLGN neurons, potentially due to the mismatch in suprathreshold and subthreshold visual inputs. In the case of binocular neurons, binocular viewing leads to even lower activity levels compared with monocular viewing through the dominant eye (binocular suppression, indicated by " $-b^{\prime \prime}$ and binocular modulation values $<1$ ). For simplicity, the model does not depict other modes of binocular modulation, such as binocular activation and complete suppression ("both only" and "suppressed" in Fig. 10).

critical-period MD (Fig. 12). These data suggest that criticalperiod visual experience is essential for the development of normal function, rather than structure, of dLGN projections to superficial layers of $\mathrm{V} 1$.

\section{Discussion}

We examined the role of early visual experience in shaping visual properties of thalamocortical inputs from dLGN to superficial layers of bV1. We demonstrate that long-term sensory deprivation (14 d of MD) during the critical period for OD plasticity leads to a number of persistent changes in dLGN inputs, including a loss of binocular dLGN afferents, binocular mismatch of tuning properties in remaining binocular inputs, and impaired binocular facilitation of both binocular and monocular dLGN responses (Fig. 13). A number of other properties, such as response strength and spatial acuity, were preserved at the level of dLGN afferents following long-term MD. Similar to thalamic inputs, V1 L4 neurons also exhibited binocularity loss and intact spatial acuity in deprived mice. However, V1 L2/3 neurons displayed both binocularity and acuity loss following MD. Longlasting changes in OD, spatial acuity, and binocular mismatch in orientation tuning have previously been observed for V1 neurons in MD models (Heimel et al., 2007; Stephany et al., 2014; Levine et al., 2017), yet it has remained uncertain whether these changes originate from V1 circuits or from thalamic inputs. Our findings establish, for the first time, that some of these MD-induced changes, persistent alterations in OD and binocular mismatch in orientation and SF tuning, are already present at the level of thalamocortical inputs; thus, these deficits may originate from thalamic circuits. On the other hand, overall SF processing is intact in dLGN inputs and V1 L4 neurons but impaired in V1 L2/3 neurons following MD. These findings provide the most direct evidence to date indicating that early binocular experience is required to properly develop binocularity in the thalamus, while it is also needed to support acuity development in cortical circuits beginning in L2/3 of V1. Thus, different stages in visual processing and potentially distinct mechanisms (Stephany et al., 2014, 2018) may be involved in the development of binocular vision versus spatial acuity in the mammalian visual system.

In many aspects, our results are in good agreement with recent functional and anatomical studies. Jaepel et al. (2017) reported that $\sim 14 \%$ of dLGN boutons in mouse V1 L1 are binocular and that OD plasticity can be induced in dLGN afferents in adult mice under enriched conditions, consistent with our findings on juvenile plasticity. Notably, however, the binocular fraction increased immediately and transiently following MD in Jaepel et al. (2017), which may reflect effects of shorter-term MD used in their study. We found that binocular boutons exhibit stronger visual responses compared with monocular ones, which is in agreement with an anatomical report that binocular dLGN cells combine inputs from a larger number of RGCs compared with monocular dLGN cells (Rompani et al., 2017). The anatomical study suggested that binocular and "combination-mode" dLGN neurons integrate inputs from many RGCs of several cell types, whereas "relay-mode" dLGN neurons receive inputs from a few RGCs of mostly one type. This model predicts that binocular neurons should possess broad tuning properties due to input diversity. 
Indeed, we found that binocular dLGN boutons are generally broader in $\mathrm{SF}$ and orientation tuning compared with monocular dLGN boutons (Figs. 5F, 9A, B).

It is currently unknown the exact locus and neural mechanisms involved in the loss of binocularity in dLGN afferents following long-term MD. Considering preservation of structural integrity of the axons and of several functional characteristics, the site of binocularity loss seems most likely to be at the retinogeniculate synapse (Fig. 13). Recent work has revealed complex mechanisms with which retinogeniculate synapses are integrated and fine-tuned quite late into development (Jaubert-Miazza et al., 2005; Thompson et al., 2016; Litvina and Chen, 2017; Rompani et al., 2017; Cheadle et al., 2018; Román Rosón et al., 2019). Accordingly, dLGN neurons undergo substantial refinement of their receptive field properties as late as third postnatal week (Tschetter et al., 2018). It is possible that the integration of multiple synaptic inputs involved in binocular development engages pruning of mismatched inputs through classic Hebbian plasticity mechanisms or other activity-dependent mechanisms (Krahe and Guido, 2011). Previously, it was demonstrated that summed thalamic inputs onto V1 neurons are already somewhat matched between the eyes before the critical period, suggesting that dLGN may help shape binocular matching in V1 neurons during development ( $\mathrm{Gu}$ and Cang, 2016). Our surprising finding that, in mismatched boutons, ipsilateral- (nondeprived) eye responses are placed at a greater disadvantage compared with contralateraleye responses hints at an intriguing possibility that ipsilateral inputs rely on contralateral inputs to guide the matching process. This is certainly in line with findings showing that the ipsilateral pathway develops later and is more vulnerable to developmental manipulations compared with the contralateral pathway (Dräger, 1978; Godement et al., 1987; Sretavan and Shatz, 1987; Gordon and Stryker, 1996; Scholl et al., 2017).

We cannot rule out the possibility that some of the binocularity loss may be due to structural loss of binocular thalamocortical projections. Our structural analysis was at a gross scale, and we did not characterize morphology of individual axon arbors. However, our finding of overall structural integrity following $14 \mathrm{~d}$ of $\mathrm{MD}$ is consistent with previous studies that also reported little to no long-lasting morphological changes in thalamocortical projections following 7 or $20 \mathrm{~d}$ of MD in mice (Antonini et al., 1999; Coleman et al., 2010). This is in stark contrast to rapid morphological changes observed in other species (Antonini and Stryker, 1993). Furthermore, our findings here focus on dLGN axons in superficial layers of $\mathrm{V} 1$, and it is unknown whether these results may be representative of all dLGN inputs. In addition, as with all axon calcium imaging studies, there exists a possibility that the findings may partially reflect local (cortical) influences on the terminals.

The most surprising finding here is that long-term criticalperiod MD leads to a loss of binocular facilitation, affecting visual responses of both binocular and monocular dLGN inputs. We found the mean binocular modulation of dLGN inputs to be 1.19 in controls and 1.04 in MD mice. Human psychophysics studies reported the benefit of both-eye viewing over monocular viewing to be $\sim \sqrt{ } 2$ (1.41) on simple visual tasks (Frisén and Lindblom, 1988). In cats and monkeys, the effects of binocular viewing in dLGN appear to be mostly suppressive (Marrocco and McClurkin, 1979; Rodieck and Dreher, 1979; Schroeder et al., 1990; Zeater et al., 2015), although recent studies conducted in awake monkeys have reported significant binocular facilitation in dLGN neurons and in V1 layer 4C neurons that receive direct thalamocortical inputs (Schroeder et al., 1990; Dougherty et al., 2019a). Before our study, others have demonstrated binocular modulation of mouse dLGN neurons, including facilitation and suppression (Zhao et al., 2013; Howarth et al., 2014). Our results extend these findings and further show that binocular modulation in dLGN is subject to long-lasting developmental perturbations. Moreover, binocular modulation of monocular dLGN inputs is correlated with response strength, such that weaker responses are binocularly facilitated while stronger responses are binocularly suppressed, suggesting homeostatic regulation of net visual drive (Mrsic-Flogel et al., 2007). Binocular dLGN boutons, however, display the opposite pattern, and their binocular modulation correlates with binocularity. A similar correlation with binocularity was reported in monkey V1 L4 cells (Dougherty et al., 2019a). Binocular suppression in dLGN may be mediated by local interneurons via lateral connections (Seabrook et al., 2013) and/or from cortical feedback. Meanwhile, dLGN binocular facilitation may arise from a direct, normally subthreshold, excitatory input from the nondominant eye (Fig. 13). Howarth et al. (2014) suggested that binocular facilitation may be mediated by corticogeniculate feedback. Future studies will be needed to uncover the neural basis underlying binocular modulation in distinct dLGN cell types and in different species (Rodieck and Dreher, 1979).

Together, our findings demonstrate that binocular integration in the early feedforward pathway from dLGN to V1 requires normal binocular experience during the critical period to develop properly. Although it is likely that binocular competition plays a role (Penn et al., 1998), the exact mechanism of action, cell types, and molecular factors involved in this developmental mechanism remain to be elucidated (Dhande and Huberman, 2014; Kerschensteiner and Guido, 2017; Miska et al., 2018). Considering our results, future studies investigating OD plasticity, binocular matching, and other types of binocular interactions will need to disambiguate relative contributions of thalamic versus cortical mechanisms (Scholl et al., 2013; La Chioma et al., 2019; Samonds et al., 2019). Strikingly, it has been demonstrated that human amblyopic observers display abnormal binocular integration in certain visual tasks (Levi et al., 1979), resulting in performance with two eyes being no better than, or even worse than, that with one eye. To assess the true significance of our findings in the context of human amblyopia, it will be important to determine whether binocular integration in the primate dLGN (Zeater et al., 2015) is also vulnerable to sensory manipulations during development. If, indeed, binocular function is first impaired in the thalamus and acuity deficits first arise from the cortex in human amblyopia, it would have critical implications for understanding of the disorder and its treatment.

\section{References}

Allen B, Spiegel DP, Thompson B, Pestilli F, Rokers B (2015) Altered white matter in early visual pathways of humans with amblyopia. Vision Res 114:48-55.

Antonini A, Stryker MP (1993) Rapid remodeling of axonal arbors in the visual cortex. Science 260:1819-1821.

Antonini A, Fagiolini M, Stryker MP (1999) Anatomical correlates of functional plasticity in mouse visual cortex. J Neurosci 19:4388-4406.

Blakemore C, Vital-Durand F (1986) Effects of visual deprivation on the development of the monkey's lateral geniculate nucleus. J Physiol 380: 493-511.

Bochner DN, Sapp RW, Adelson JD, Zhang S, Lee H, Djurisic M, Syken J, Dan Y, Shatz CJ (2014) Blocking PirB up-regulates spines and functional synapses to unlock visual cortical plasticity and facilitate recovery from amblyopia. Sci Transl Med 6:258ra140.

Casagrande VA, Boyd JD (1996) The neural architecture of binocular vision. Eye 10:153-160. 
Cheadle L, Tzeng CP, Kalish BT, Harmin DA, Rivera S, Ling E, Nagy MA, Hrvatin S, Hu L, Stroud H, Burkly LC, Chen C, Greenberg ME (2018) Visual experience-dependent expression of Fn14 is required for retinogeniculate refinement. Neuron 99:525-539.e10.

Chen TW, Wardill TJ, Sun Y, Pulver SR, Renninger SL, Baohan A, Schreiter ER, Kerr RA, Orger MB, Jayaraman V, Looger LL, Svoboda K, Kim DS (2013) Ultrasensitive fluorescent proteins for imaging neuronal activity. Nature 499:295-300.

Coleman JE, Nahmani M, Gavornik JP, Haslinger R, Heynen AJ, Erisir A, Bear MF (2010) Rapid structural remodeling of thalamocortical synapses parallels experience-dependent functional plasticity in mouse primary visual cortex. J Neurosci 30:9670-9682.

Cruz-Martín A, El-Danaf RN, Osakada F, Sriram B, Dhande OS, Nguyen PL, Callaway EM, Ghosh A, Huberman AD (2014) A dedicated circuit links direction-selective retinal ganglion cells to the primary visual cortex. Nature 507:358-361.

Davis MF, Figueroa Velez DX, Guevarra RP, Yang MC, Habeeb M, Carathedathu MC, Gandhi SP (2015) Inhibitory neuron transplantation into adult visual cortex creates a new critical period that rescues impaired vision. Neuron 86:1055-1066.

Daw NW, Fox K, Sato H, Czepita D (1992) Critical period for monocular deprivation in the cat visual cortex. J Neurophysiol 67:197-202.

Dhande OS, Huberman AD (2014) Retinal ganglion cell maps in the brain: implications for visual processing. Curr Opin Neurobiol 24:133-142.

Dougherty K, Cox MA, Westerberg JA, Maier A (2019a) Binocular modulation of monocular V1 neurons. Curr Biol 29:381-391.e4.

Dougherty K, Schmid MC, Maier A (2019b) Binocular response modulation in the lateral geniculate nucleus. J Comp Neurol 527:522-534.

Dräger UC (1978) Observations on monocular deprivation in mice. J Neurophysiol 41:28-42.

Dubbs A, Guevara J, Yuste R (2016) moco: fast motion correction for calcium imaging. Front Neuroinform 160:269-290.

Duffy KR, Holman KD, Mitchell DE (2014) Shrinkage of X cells in the lateral geniculate nucleus after monocular deprivation revealed by FoxP2 labeling. Vis Neurosci 31:253-261.

Durand S, Iyer R, Mizuseki K, de Vries S, Mihalas S, Reid RC (2016) A comparison of visual response properties in the lateral geniculate nucleus and primary visual cortex of awake and anesthetized mice. J Neurosci 36:12144-12156.

Feng L, Zhao T, Kim J (2015) neuTube 1.0: a new design for efficient neuron reconstruction software based on the SWC format. eNeuro 2:ENEURO. 0049-14.2014.

Frisén L, Lindblom B (1988) Binocular summation in humans: evidence for a hierarchic model. J Physiol 402:773-782.

Godement P, Vanselow J, Thanos S, Bonhoeffer F (1987) A study in developing visual systems with a new method of staining neurones and their processes in fixed tissue. Development 101:697-713.

Gordon JA, Stryker MP (1996) Experience-dependent plasticity of binocular responses in the primary visual cortex of the mouse. J Neurosci 16: $3274-3286$.

Gu Y, Cang J (2016) Binocular matching of thalamocortical and intracortical circuits in the mouse visual cortex. Elife 5:e22032.

Heimel JA, Hartman RJ, Hermans JM, Levelt CN (2007) Screening mouse vision with intrinsic signal optical imaging. Eur J Neurosci 25:795-804.

Herzog E, Bellenchi GC, Gras C, Bernard V, Ravassard P, Bedet C, Gasnier B, Giros B, El Mestikawy S (2001) The existence of a second vesicular glutamate transporter specifies subpopulations of glutamatergic neurons. J Neurosci 21:RC181.

Hess RF, Thompson B, Gole G, Mullen KT (2009) Deficient responses from the lateral geniculate nucleus in humans with amblyopia. Eur J Neurosci 29:1064-1070.

Howarth M, Walmsley L, Brown TM (2014) Binocular integration in the mouse lateral geniculate nuclei. Curr Biol 24:1241-1247.

Jaepel J, Hübener M, Bonhoeffer T, Rose T (2017) Lateral geniculate neurons projecting to primary visual cortex show ocular dominance plasticity in adult mice. Nat Neurosci 20:1708-1714.

Jaubert-Miazza L, Green E, Lo FS, Bui K, Mills J, Guido W (2005) Structural and functional composition of the developing retinogeniculate pathway in the mouse. Vis Neurosci 22:661-676.

Kalatsky VA, Stryker MP (2003) New paradigm for optical imaging: temporally encoded maps of intrinsic signal. Neuron 38:529-545.

Kameyama K, Sohya K, Ebina T, Fukuda A, Yanagawa Y, Tsumoto T (2010)
Difference in binocularity and ocular dominance plasticity between GABAergic and excitatory cortical neurons. J Neurosci 30:1551-1559.

Kaneko M, Stryker MP (2014) Sensory experience during locomotion promotes recovery of function in adult visual cortex. Elife 3:e02798.

Kerlin AM, Andermann ML, Berezovskii VK, Reid RC (2010) Broadly tuned response properties of diverse inhibitory neuron subtypes in mouse visual cortex. Neuron 67:858-871.

Kerschensteiner D, Guido W (2017) Organization of the dorsal lateral geniculate nucleus in the mouse. Vis Neurosci 34:e008.

Kondo S, Ohki K (2016) Laminar differences in the orientation selectivity of geniculate afferents in mouse primary visual cortex. Nat Neurosci 19:316-319.

Krahe TE, Guido W (2011) Homeostatic plasticity in the visual thalamus by monocular deprivation. J Neurosci 31:6842-6849.

La Chioma A, Bonhoeffer T, Hübener M (2019) Area-specific mapping of binocular disparity across mouse visual cortex. Curr Biol 29:29542960.e5.

Levi DM (2013) Linking assumptions in amblyopia. Vis Neurosci 30: 277-287.

Levi DM, Harwerth RS, Manny RE (1979) Suprathreshold spatial frequency detection and binocular interaction in strabismic and anisometropic amblyopia. Invest Ophthalmol Vis Sci 18:714-725.

Levine JN, Chen H, Gu Y, Cang J (2017) Environmental enrichment rescues binocular matching of orientation preference in the mouse visual cortex. J Neurosci 37:5822-5833.

Levitt JB, Schumer RA, Sherman SM, Spear PD, Movshon JA (2001) Visual response properties of neurons in the LGN of normally reared and visually deprived macaque monkeys. J Neurophysiol 85:2111-2129.

Litvina EY, Chen C (2017) Functional convergence at the retinogeniculate synapse. Neuron 96:330-338.e5.

Madisen L, Zwingman TA, Sunkin SM, Oh SW, Zariwala HA, Gu H, Ng LL, Palmiter RD, Hawrylycz MJ, Jones AR, Lein ES, Zeng H (2010) A robust and high-throughput cre reporting and characterization system for the whole mouse brain. Nat Neurosci 13:133-140.

Marrocco RT, McClurkin JW (1979) Binocular interaction in the lateral geniculate nucleus of the monkey. Brain Res 168:633-637.

Marshel JH, Kaye AP, Nauhaus I, Callaway EM (2012) Anterior-posterior direction opponency in the superficial mouse lateral geniculate nucleus. Neuron 76:713-720.

Miska NJ, Richter LM, Cary BA, Gjorgjieva J, Turrigiano GG (2018) Sensory experience inversely regulates feedforward and feedback excitationinhibition ratio in rodent visual cortex. Elife 7:e38846.

Morgenstern NA, Bourg J, Petreanu L (2016) Multilaminar networks of cortical neurons integrate common inputs from sensory thalamus. Nat Neurosci 19:1034-1040.

Movshon JA, Van Sluyters RC (1981) Visual neural development. Annu Rev Psychol 32:477-522.

Mrsic-Flogel TD, Hofer SB, Ohki K, Reid RC, Bonhoeffer T, Hübener M (2007) Homeostatic regulation of eye-specific responses in visual cortex during ocular dominance plasticity. Neuron 54:961-972.

Penn AA, Riquelme PA, Feller MB, Shatz CJ (1998) Competition in retinogeniculate patterning driven by spontaneous activity. Science 279:21082112 .

Piscopo DM, El-Danaf RN, Huberman AD, Niell CM (2013) Diverse visual features encoded in mouse lateral geniculate nucleus. J Neurosci 33: 4642-4656.

Pizzorusso T, Medini P, Landi S, Baldini S, Berardi N, Maffei L (2006) Structural and functional recovery from early monocular deprivation in adult rats. Proc Natl Acad Sci U S A 103:8517-8522.

Prusky GT, Douglas RM (2003) Developmental plasticity of mouse visual acuity. Eur J Neurosci 17:167-173.

Rodieck RW, Dreher B (1979) Visual suppression from nondominant eye in the lateral geniculate nucleus: a comparison of cat and monkey. Exp Brain Res 35:465-477.

Román Rosón M, Bauer Y, Kotkat AH, Berens P, Euler T, Busse L (2019) Mouse dLGN receives functional input from a diverse population of retinal ganglion cells with limited convergence. Neuron 102:462-476.e8.

Rompani SB, Müllner FE, Wanner A, Zhang C, Roth CN, Yonehara K, Roska B (2017) Different modes of visual integration in the lateral geniculate nucleus revealed by single-cell-initiated transsynaptic tracing. Neuron 93:767-776.e6.

Roth MM, Dahmen JC, Muir DR, Imhof F, Martini FJ, Hofer SB (2016) 
Thalamic nuclei convey diverse contextual information to layer 1 of visual cortex. Nat Neurosci 19:299-307.

Salinas KJ, Figueroa Velez DX, Zeitoun JH, Kim H, Gandhi SP (2017) Contralateral bias of high spatial frequency tuning and cardinal direction selectivity in mouse visual cortex. J Neurosci 37:10125-10138.

Samonds JM, Choi V, Priebe NJ (2019) Mice discriminate stereoscopic surfaces without fixating in depth. J Neurosci 39:8024-8037.

Scholl B, Burge J, Priebe NJ (2013) Binocular integration and disparity selectivity in mouse primary visual cortex. J Neurophysiol 109:3013-3024.

Scholl B, Pattadkal JJ, Priebe NJ (2017) Binocular disparity selectivity weakened after monocular deprivation in mouse V1. J Neurosci 37:6517-6526.

Schroeder CE, Tenke CE, Arezzo JC, Vaughan HG Jr (1990) Binocularity in the lateral geniculate nucleus of the alert macaque. Brain Res 521:303-310.

Seabrook TA, Krahe TE, Govindaiah G, Guido W (2013) Interneurons in the mouse visual thalamus maintain a high degree of retinal convergence throughout postnatal development. Neural Dev 8:24.

Shatz CJ, Stryker MP (1978) Ocular dominance in layer IV of the cat's visual cortex and the effects of monocular deprivation. J Physiol 281:267-283.

Sherman SM, Hoffmann KP, Stone J (1972) Loss of a specific cell type from the dorsal lateral geniculate nucleus in visually deprived cats. J Neurophysiol 35:532-541.

Sommeijer JP, Ahmadlou M, Saiepour MH, Seignette K, Min R, Heimel JA, Levelt CN (2017) Thalamic inhibition regulates critical-period plasticity in visual cortex and thalamus. Nat Neurosci 20:1715-1721.

Sretavan DW, Shatz CJ (1987) Axon trajectories and pattern of terminal arborization during the prenatal development of the cat's retinogeniculate pathway. J Comp Neurol 255:386-400.

Stephany CÉ, Chan LL, Parivash SN, Dorton HM, Piechowicz M, Qiu S, McGee AW (2014) Plasticity of binocularity and visual acuity are differentially limited by nogo receptor. J Neurosci 34:11631-11640.

Stephany CÉ, Ma X, Dorton HM, Wu J, Solomon AM, Frantz MG, Qiu S,
McGee AW (2018) Distinct circuits for recovery of eye dominance and acuity in murine amblyopia. Curr Biol 28:1914-1923.e5.

Sun W, Tan Z, Mensh BD, Ji N (2016) Thalamus provides layer 4 of primary visual cortex with orientation- and direction-tuned inputs. Nat Neurosci 19:308-315.

Thompson AD, Picard N, Min L, Fagiolini M, Chen C (2016) Cortical feedback regulates feedforward retinogeniculate refinement. Neuron 91: 1021-1033.

Trachtenberg JT, Trepel C, Stryker MP (2000) Rapid extragranual plasticity in the absence of thalamocortical plasticity in the developing primary visual cortex. Science 287:2029-2032.

Tschetter WW, Govindaiah G, Etherington IM, Guido W, Niell CM (2018) Refinement of spatial receptive fields in the developing mouse LGN is coordinated with excitatory and inhibitory remodeling. J Neurosci 38: 4531-4542.

Wang BS, Sarnaik R, Cang J (2010) Critical period plasticity matches binocular orientation preference in the visual cortex. Neuron 65:246-256.

Wekselblatt JB, Flister ED, Piscopo DM, Niell CM (2016) Large-scale imaging of cortical dynamics during sensory perception and behavior. J Neurophysiol 115:2852-2866.

Wiesel TN, Hubel DH (1963a) Single-cell responses in striate cortex of kittens deprived of vision in one eye. J Neurophysiol 26:1003-1017.

Wiesel TN, Hubel DH (1963b) Effects of visual deprivation on morphology and physiology of cells in the cat's lateral geniculate body. J Neurophysiol 26:978-993.

Zeater N, Cheong SK, Solomon SG, Dreher B, Martin PR (2015) Binocular visual responses in the primate lateral geniculate nucleus. Curr Biol 25:3190-3195.

Zhao X, Liu M, Cang J (2013) Sublinear binocular integration preserves orientation selectivity in mouse visual cortex. Nat Commun 4:2088. 